\title{
Spectral Analysis of a Quantum System with a Double Line Singular Interaction
}

\author{
by \\ Sylwia KondeJ and David KREJČIŘÍK
}

\begin{abstract}
We consider a non-relativistic quantum particle interacting with a singular potential supported by two parallel straight lines in the plane. We locate the essential spectrum under the hypothesis that the interaction asymptotically approaches a constant value, and find conditions which guarantee either the existence of discrete eigenvalues or Hardytype inequalities. For a class of our models admitting mirror symmetry, we also establish the existence of embedded eigenvalues and show that they turn into resonances after introducing a small perturbation.
\end{abstract}

2010 Mathematics Subject Classification: 81Q10, 35P15, 35J10, 35B34, 35B25.

Keywords: Schrödinger operator, singular perturbation, spectral analysis, Hardy inequality, resonance.

\section{$\S 1$. Introduction}

The problem we study in this paper belongs to the line of research often called singular perturbations of Schrödinger operators. Let us consider a non-relativistic quantum particle confined to a semiconductor structure $\Sigma \subset \mathbb{R}^{3}$. Suppose the particle has a possibility of tunnelling, so the whole $\mathbb{R}^{3}$ forms the configuration space. On the other hand, if the device $\Sigma$ is narrow in a sense, we can make an idealization and assume that $\Sigma$ is a set of lower dimension, for example, a surface, a curve or dots in $\mathbb{R}^{3}$. Consequently, we come to the model of quantum systems with potential interaction supported by a null set. The interaction can vary on $\Sigma$; let a function $V: \Sigma \rightarrow \mathbb{R}$ denote the potential strength. Then the Hamiltonian of

Communicated by H. Okamoto. Received July 4, 2012. Revised February 26, 2013, and April 19, 2013.

S. Kondej: Institute of Physics, University of Zielona Góra,

Szafrana 4a, 65246 Zielona Góra, Poland;

e-mail: skondej@proton.if .uz.zgora.pl

D. Krejčiř́ík: Department of Theoretical Physics, Nuclear Physics Institute ASCR,

25068 Rež, Czech Republic;

e-mail: krejcirik@ujf.cas.cz 
such a quantum system can be symbolically written as

$$
-\Delta+V \delta(\cdot-\Sigma)
$$

where $-\Delta$ denotes the Laplace operator in $L^{2}\left(\mathbb{R}^{3}\right)$ and $\delta$ is the Dirac delta function. In view of singular interactions with translational symmetry, it also makes sense to consider one- and two-dimensional analogues of (1.1).

There are a lot of papers devoted to analysing the relation between the geometry of $\Sigma$ and spectral properties of the Hamiltonian with delta interactions of constant strength $[6,11,12,13,15,16]$; we also refer to the monographs $[2,3]$ with many references. Delta-type potentials supported on infinite curves and surfaces are used in particular for mathematical modelling of leaky quantum wires and graphs $[9,4]$.

The simplest known model belonging to the class described by (1.1) is given by $N$ quantum dots in one dimension [2, Chap. I.3, II.2]. Then $\Sigma:=\left\{x_{i}\right\}_{i=0}^{N-1}$ and $V\left(x_{i}\right):=-\alpha_{i} \in \mathbb{R}$. For one point interaction (i.e. $N=1, \alpha_{0}=: \alpha$ ) the system has one negative eigenvalue $-\alpha^{2} / 4$ if, and only if, $\alpha$ is positive. In the case of two point interactions of equal strength (i.e. $N=2, \alpha_{0}=\alpha_{1}=: \alpha$ ) at distance $2 a$ from each other, there is one negative eigenvalue $\xi_{0}$ if, and only if, $0<\alpha a \leq 1$ or two eigenvalues $\xi_{0}<\xi_{1}$ whenever $\alpha a>1$.

The problem we discuss in this paper can be considered as a generalization of the two quantum dots in two respects. First, our model is two-dimensional, with the set $\Sigma$ being one-dimensional. Second, the generalized geometry enables us to consider potentials $V$ of variable strength. More specifically, we consider the singular set $\Sigma$ composed of two infinite lines

$$
\Sigma:=\Sigma_{-} \cup \Sigma_{+} \quad \text { with } \quad \Sigma_{ \pm}:=\mathbb{R} \times\{ \pm a\}
$$

in $\mathbb{R}^{2}$ and

$$
V(x):= \begin{cases}-\alpha+V_{+}(x) & \text { if } x \in \Sigma_{+}, \\ -\alpha+V_{-}(x) & \text { if } x \in \Sigma_{-},\end{cases}
$$

with $V_{ \pm}: \Sigma_{ \pm} \rightarrow \mathbb{R}$ and $\alpha>0$. In the physical setting described above, the negative part $-\alpha$ of $V$ models the confinement of the particle to $\Sigma$, while $V_{ \pm}$can be thought of as a perturbation.

Our first aim is to find a self-adjoint realization in $L^{2}\left(\mathbb{R}^{2}\right)$ of (1.1) with (1.2)(1.3). We will apply the form-sum method and the resulting operator will be called $H_{\alpha, V_{+}, V_{-}}$. Note that the delta potential in our model does not vanish at infinity even if $V_{ \pm}$do (just because $\alpha$ is assumed to be positive). This means that we may expect that the essential spectrum of $H_{\alpha, V_{+}, V_{-}}$will differ from the spectrum of the 
free Hamiltonian in $\mathbb{R}^{2}$. In our setting, the role of the unperturbed Hamiltonian is played by $H_{\alpha, 0,0}$.

For the unperturbed Hamiltonian, translational symmetry allows us to decompose the operator as follows:

$$
H_{\alpha, 0,0} \simeq\left(-\Delta^{\mathbb{R}}\right) \otimes 1+1 \otimes\left(-\Delta_{\alpha}^{\mathbb{R}}\right) \quad \text { on } L^{2}(\mathbb{R}) \otimes L^{2}(\mathbb{R}),
$$

where $-\Delta^{\mathbb{R}}$ is the free one-dimensional Hamiltonian and $-\Delta_{\alpha}^{\mathbb{R}}$ governs the aforementioned one-dimensional system with two point interactions. The non-negative semi-axis is the spectrum of $-\Delta^{\mathbb{R}}$. On the other hand, as already mentioned, the spectrum of $-\Delta_{\alpha}^{\mathbb{R}}$ is

$$
\sigma\left(-\Delta_{\alpha}^{\mathbb{R}}\right)=\sigma_{\text {disc }}\left(-\Delta_{\alpha}^{\mathbb{R}}\right) \cup[0, \infty)
$$

with the negative eigenvalues (cf. Lemma 2.3 and Figure 1)

$$
\sigma_{\text {disc }}\left(-\Delta_{\alpha}^{\mathbb{R}}\right)= \begin{cases}\left\{\xi_{0}\right\} & \text { if } 0<\alpha a \leq 1 \\ \left\{\xi_{0}, \xi_{1}\right\} & \text { if } \alpha a>1\end{cases}
$$

(the discrete spectrum is empty in the other situations, which are excluded here by the assumption $\alpha>0$ ). Recalling the ordering $\xi_{0}<\xi_{1}$ (if the latter eigenvalue exists), we conclude that (irrespective of the value of $\alpha a$ )

$$
\sigma\left(H_{\alpha, 0,0}\right)=\sigma_{\mathrm{ess}}\left(H_{\alpha, 0,0}\right)=\left[\xi_{0}, \infty\right) .
$$

The main results of the paper can be formulated as follows.

- Definition of the Hamiltonian and its resolvent. The definition of the Hamiltonian $H_{\alpha, V_{+}, V_{-}}$by use of the form-sum method is given in Section 2. We also derive a Krein-like formula for the resolvent of $H_{\alpha, V_{+}, V_{-}}$as a tool for further discussion.

- Essential spectrum. In Section 3 we find a weak condition guaranteeing the stability of the essential spectrum of $H_{\alpha, V_{+}, V_{-}}$with respect to $H_{\alpha, 0,0}$. We show that if $V_{ \pm}$vanish at infinity then

$$
\sigma_{\mathrm{ess}}\left(H_{\alpha, V_{+}, V_{-}}\right)=\sigma_{\mathrm{ess}}\left(H_{\alpha, 0,0}\right)=\left[\xi_{0}, \infty\right)
$$

The strategy of our proof is as follows. Using a Neumann bracketing argument together with the minimax principle, we get inf $\sigma_{\text {ess }}\left(H_{\alpha, V_{+}, V_{-}}\right) \geq \xi_{0}$. The opposite inclusion $\sigma_{\text {ess }}\left(H_{\alpha, V_{+}, V_{-}}\right) \supseteq\left[\xi_{0}, \infty\right)$ is obtained by applying the Weyl criterion adapted to sesquilinear forms.

- Discrete and embedded eigenvalues. The point spectrum is investigated in Section 4 . We show that the bottom of the spectrum of $H_{\alpha, V_{+}, V_{-}}$starts below $\xi_{0}$ 
provided that the sum $V_{+}+V_{-}$is negative in an integral sense. Assuming additionally that $V_{ \pm}$vanish at infinity and combining this with the previous result on the essential spectrum, we therefore obtain

$$
\sigma_{\text {disc }}\left(H_{\alpha, V_{+}, V_{-}}\right) \neq \emptyset \text {. }
$$

The proof is based on finding a suitable test function in the variational definition of the spectral threshold. We also find conditions which guarantee the existence of embedded eigenvalues in the system with mirror symmetry, i.e. when $V_{+}=V_{-}$.

- Hardy inequalities. The case of repulsive singular potentials, i.e. $V_{ \pm} \geq 0$, is studied in Section 5. In order to quantify the repulsive character of the singular potential, we derive Hardy-type inequalities

$$
H_{\alpha, V_{+}, V_{-}}-\xi_{0} \geq \varrho
$$

in the form sense, where $\varrho: \mathbb{R} \rightarrow[0, \infty)$ is not identically zero. The functional inequality (1.8) is useful in the study of spectral stability of $H_{\alpha, V_{+}, V_{-}}$; indeed, it determines a class of potentials which can be added to our system without producing any spectrum below $\xi_{0}$.

- Resonances. Finally, in Section 6 we show that breaking the mirror symmetry by introducing a "perturbant" function $V_{p}$ on one of the lines, for example

$$
V_{+}=V_{0}+\epsilon V_{p}, \quad V_{-}=V_{0},
$$

leads to resonances. These resonances are localized near the original embedded eigenvalues $\nu_{k}$ appearing when $\epsilon=0$. More precisely, they are determined by poles of the resolvent which take the form

$$
z_{k}=\nu_{k}+\mu_{k}(\epsilon)+i v_{k}(\epsilon)
$$

where $\mu_{k}(\epsilon)=a_{k} \epsilon+\mathcal{O}\left(\epsilon^{2}\right)$ with $a_{k}$ corresponding to the first order perturbation term and $v_{k}(\epsilon)=b_{k} \mathcal{O}\left(\epsilon^{2}\right)$ where $b_{k}<0$ establishes the Fermi golden rule.

Let us conclude this introductory section by pointing out some special notations frequently used throughout the paper. We abbreviate $L^{2}:=L^{2}\left(\mathbb{R}^{2}\right)$ and $L_{ \pm}^{2}:=L^{2}(\mathbb{R} \times\{ \pm a\})$. We also briefly write $W^{n, 2}:=W^{n, 2}\left(\mathbb{R}^{2}\right)$ for the corresponding Sobolev spaces. The inner product and norm in $L^{2}$ are denoted by $(\cdot, \cdot)$ and $\|\cdot\|$, respectively. Given a self-adjoint operator $H$, the symbols $\sigma_{\iota}(H)$ with $\iota \in\{$ ess, ac, sc, p, disc $\}$ denote, respectively, the essential, absolutely continuous, singularly continuous, point and discrete spectrum of $H$. We use the symbol 1 to denote the identity operators acting in various Hilbert spaces. 


\section{§2. The Hamiltonian and its resolvent}

Let $V_{+}$and $V_{-}$be two real-valued functions from $L^{\infty}(\mathbb{R})$. Given a positive number $a$, we also denote by $V_{+}$and $V_{-}$the functions $V_{+} \otimes 1$ and $V_{-} \otimes 1$ on $\mathbb{R} \times\{+a\}$ and $\mathbb{R} \times\{-a\}$, respectively. Finally, let $\alpha$ be a positive constant.

\section{$\S 2.1$. The self-adjoint realization of the Hamiltonian}

Let us consider the quadratic form

$$
\begin{aligned}
\mathcal{E}_{\alpha, V_{+}, V_{-}}[\psi] & :=\int_{\mathbb{R}^{2}}|\nabla \psi|^{2}+\int_{\mathbb{R} \times\{+a\}}\left(V_{+}-\alpha\right)\left|I_{+} \psi\right|^{2}+\int_{\mathbb{R} \times\{-a\}}\left(V_{-}-\alpha\right)\left|I_{-} \psi\right|^{2}, \\
\mathfrak{D}\left(\mathcal{E}_{\alpha, V_{+}, V_{-}}\right) & :=W^{1,2} .
\end{aligned}
$$

Here $I_{ \pm}$are the trace operators associated with the Sobolev embedding $W^{1,2} \hookrightarrow$ $L_{ \pm}^{2}$. The corresponding sesquilinear form will be denoted by $\mathcal{E}_{\alpha, V_{+}, V_{-}}(\cdot, \cdot)$.

The form $\mathcal{E}_{\alpha, V_{+}, V_{-}}$is clearly densely defined and symmetric. Moreover, the boundary integrals can be shown to be a relatively bounded perturbation of the form $\mathcal{E}_{0,0,0}$ with relative bound less than 1 . This is a consequence of the boundedness of $V_{ \pm}$and the following result.

Lemma 2.1. For every $\psi \in W^{1,2}$ and $\epsilon \in(0,1)$, we have

$$
\left\|I_{ \pm} \psi\right\|_{L_{ \pm}^{2}}^{2} \leq \frac{1}{\epsilon}\|\psi\|_{L^{2}}^{2}+\epsilon\left\|\partial_{2} \psi\right\|_{L^{2}}^{2} .
$$

Proof. For every $\epsilon \in(0,1)$ and $\psi \in C_{0}^{\infty}\left(\mathbb{R}^{2}\right)$, we have the bound

$$
\begin{aligned}
\left|\psi\left(x_{1}, \pm a\right)\right|^{2} & =\int_{-\infty}^{ \pm a} \partial_{2}|\psi|^{2}\left(x_{1}, x_{2}\right) \mathrm{d} x_{2}=2 \Re \int_{-\infty}^{ \pm a} \overline{\psi\left(x_{1}, x_{2}\right)} \partial_{2} \psi\left(x_{1}, x_{2}\right) \mathrm{d} x_{2} \\
& \leq \frac{1}{\epsilon} \int_{\mathbb{R}}\left|\psi\left(x_{1}, x_{2}\right)\right|^{2} \mathrm{~d} x_{2}+\epsilon \int_{\mathbb{R}}\left|\partial_{2} \psi\left(x_{1}, x_{2}\right)\right|^{2} \mathrm{~d} x_{2} .
\end{aligned}
$$

Integrating over $x_{1}$, we therefore get (2.9) for $\psi \in C_{0}^{\infty}\left(\mathbb{R}^{2}\right)$. By density, the inequality extends to $W^{1,2}$.

Remark 2.2 (Relation to the generalized Kato class). Inequality (2.9) represents a quantification of the embedding $W^{1,2} \hookrightarrow L_{ \pm}^{2}$. If the support of the singular potential has a more complicated geometry we can derive a generalization of (2.9). Consider a Radon measure $\mu$ on $\mathbb{R}^{2}$ with support on a $C^{1}$ curve (finite or infinite) without self-intersections and "near-self-intersections" (see [5, Sec. 4] for precise assumptions). Such a measure belongs to the generalized Kato class (cf. [5, Thm. 4.1]), and consequently for any $a>0$ there exists $b>0$ such that

$$
\int_{\mathbb{R}^{2}}|\psi|^{2} \mathrm{~d} \mu \leq b\|\psi\|_{L^{2}}^{2}+a\|\nabla \psi\|_{L^{2}}^{2} \quad \text { for every } \psi \in W^{1,2} .
$$


Since $\mathcal{E}_{0,0,0}$ is clearly closed and non-negative (it is in fact associated with the free Hamiltonian in $\mathbb{R}^{2}$ ), it follows from the KLMN theorem [25, Thm. X.17] together with Lemma 2.1 that $\mathcal{E}_{\alpha, V_{+}, V_{-}}$is closed and bounded from below. Consequently, there exists a unique bounded-from-below self-adjoint operator $H_{\alpha, V_{+}, V_{-}}$ in $L^{2}$ which is associated with $\mathcal{E}_{\alpha, V_{+}, V_{-}}$. (Notice that the sign of $\alpha$ plays no role in the definition of $H_{\alpha, V_{+}, V_{-}}$)

Finally, let us note that $H_{\alpha, V_{+}, V_{-}}$is indeed a natural realization of the formal expression (1.1) with (1.2)-(1.3). As a matter of fact, our form $\mathcal{E}_{\alpha, V_{+}, V_{-}}$represents a closed extension of the form associated with the expression (1.1) initially considered as acting on smooth functions rapidly decaying at the infinity of $\mathbb{R}^{2}$.

\section{§2.2. The transverse Hamiltonian}

Recall the decomposition (1.4) for the unperturbed Hamiltonian $H_{\alpha, 0,0}$. Here the "transverse" operator $-\Delta_{\alpha}^{\mathbb{R}}$ is associated with the form

$$
\varepsilon_{\alpha}[\phi]:=\int_{\mathbb{R}}\left|\phi^{\prime}\right|^{2}-\alpha|\phi(+a)|^{2}-\alpha|\phi(-a)|^{2}, \quad \mathfrak{D}\left(\varepsilon_{\alpha}\right):=W^{1,2}(\mathbb{R}) .
$$

Note that the boundary values have a meaning in view of the embedding $W^{1,2}(\mathbb{R})$ $\hookrightarrow C^{0}(\mathbb{R})$. It is easy to verify that $\mathfrak{D}\left(-\Delta_{\alpha}^{\mathbb{R}}\right)$ consists of all functions $\psi \in W^{1,2}(\mathbb{R}) \cap$ $W^{2,2}(\mathbb{R} \backslash\{-a,+a\})$ satisfying the interface conditions

$$
\psi^{\prime}( \pm a+0)-\psi^{\prime}( \pm a-0)=-\alpha \psi( \pm a)
$$

where $\psi^{\prime}(a \pm 0):=\lim _{\epsilon \rightarrow 0^{+}} \psi^{\prime}(a \pm \epsilon)$, and that $-\Delta_{\alpha}^{\mathbb{R}} \psi=-\psi^{\prime \prime}$ on $\mathbb{R} \backslash\{-a,+a\}$. An alternative way of introducing $-\Delta_{\alpha}^{\mathbb{R}}$ is via extension theory [2, Chap. II.2].

Due to [2, Thm. 2.1.3], we have $\sigma_{\text {ess }}\left(-\Delta_{\alpha}^{\mathbb{R}}\right)=\sigma_{\text {ac }}\left(-\Delta_{\alpha}^{\mathbb{R}}\right)=[0, \infty)$. The structure of the discrete spectrum depends on the value of $\alpha a$.

Lemma 2.3. The operator $-\Delta_{\alpha}^{\mathbb{R}}$ has exactly two negative eigenvalues if $\alpha a>1$ and exactly one negative eigenvalue if $0<\alpha a \leq 1$.

Proof. Let $\kappa>0$. Solving the eigenvalue problem $-\psi^{\prime \prime}=-\kappa^{2} \psi$ on $\mathbb{R} \backslash\{-a,+a\}$ in terms of exponential functions decaying at infinity and using the continuity of $\psi$ and (2.10), it is straightforward to see that the algebraic equation

$$
\frac{\alpha^{2}}{4} \mathrm{e}^{-4 \kappa a}=\left(\kappa-\frac{\alpha}{2}\right)^{2}
$$

represents a sufficient and necessary condition for $-\kappa^{2} \in \sigma_{\mathrm{p}}\left(-\Delta_{\alpha}^{\mathbb{R}}\right)$. (Alternatively, one could directly use [2, eq. (2.1.33)].) Clearly, equation (2.11) is equivalent to 
$g_{1}(\kappa)-g_{2}(\kappa)=0$ with

$$
g_{1}(\kappa):=\frac{\alpha}{2} \mathrm{e}^{-2 \kappa a} \quad \text { and } \quad g_{2}(\kappa):=\left|\kappa-\frac{\alpha}{2}\right| .
$$

Since the exponential function $g_{1}$ is positive and decreasing and $g_{2}$ is increasing in $(\alpha / 2, \infty)$ with $g_{2}(\alpha / 2)=0$, there exists exactly one solution $\kappa_{0}>\alpha / 2$ of $(2.11)$ for any $\alpha>0$. Since $g_{1}$ is strictly convex and $g_{1}(0)=g_{2}(0)$, and since the graph of $g_{2}$ is a straight line for $\kappa \in(0, \alpha / 2)$ with $g_{2}^{\prime}(0)=-1$, the existence of another (at most one) solution $\kappa_{1} \in(0, \alpha / 2)$ is determined by the derivative of $g_{1}$ at 0 . Obviously, $g_{1}^{\prime}(0) \geq-1$ if, and only if, $\alpha a \leq 1$. We set $\xi_{0}:=-\kappa_{0}^{2}$ and $\xi_{1}:=-\kappa_{1}^{2}$ (if the latter value exists).

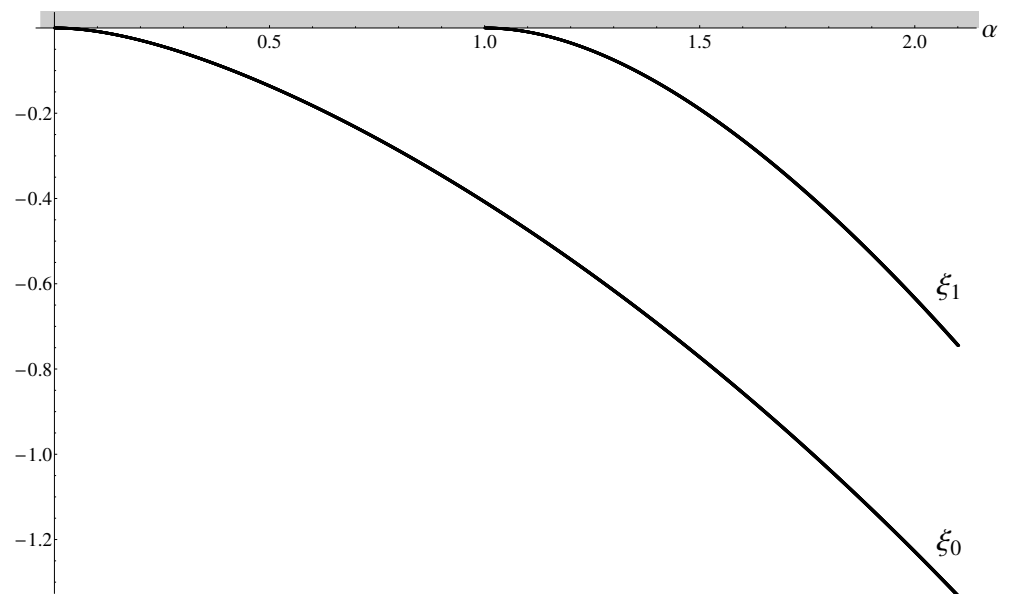

Figure 1. Emergence of the discrete eigenvalues $\xi_{0}, \xi_{1}$ of $-\Delta_{\alpha}^{\mathbb{R}}$ from the essential spectrum as $\alpha$ increases for $a=1$.

We refer to Figure 1 for the dependence of the discrete spectrum on $\alpha a$. The eigenfunctions corresponding to $\xi_{0}$ and $\xi_{1}$ will be denoted by $\phi_{0}$ and $\phi_{1}$, respectively. For notational purposes, it will become convenient to introduce the index set

$$
\mathcal{N}:= \begin{cases}\{0\} & \text { if } \alpha a \leq 1 \\ \{0,1\} & \text { if } \alpha a>1\end{cases}
$$

Due to the symmetry of the system, $\phi_{0}$ is even and $\phi_{1}$ is odd. Moreover, $\phi_{0}$ can be chosen positive [2, Thm. 2.1.3]. 


\section{§2.3. A lower-bound Hamiltonian}

In this subsection we derive an auxiliary result we shall use several times. It is based on an idea used in a similar context in [17].

Taking into account the structure of $\mathcal{E}_{\alpha, V_{+}, V_{-}}$, let us define

$$
\tilde{\lambda}\left(v_{+}, v_{-}\right):=\inf _{\phi \in W^{1,2}(\mathbb{R}) \backslash\{0\}} \frac{\varepsilon_{\alpha}[\phi]+v_{+}|\phi(+a)|^{2}+v_{-}|\phi(-a)|^{2}}{\|\phi\|_{L^{2}(\mathbb{R})}^{2}}-\xi_{0}
$$

for any real constants $v_{+}$and $v_{-}$. The number $\tilde{\lambda}\left(v_{+}, v_{-}\right)$is the lowest eigenvalue of the operator $(1.1)$ on $L^{2}(\mathbb{R})$ shifted by $\xi_{0}$, subject to two point interactions of strength $v_{+}-\alpha$ and $v_{-}-\alpha$ and at distance $2 a$ from each other.

It is clear that $\tilde{\lambda}\left(v_{+}, v_{-}\right)$is a continuous and non-decreasing function of both $v_{+}$and $v_{-}$. The symmetry relation $\tilde{\lambda}\left(v_{+}, v_{-}\right)=\tilde{\lambda}\left(v_{-}, v_{+}\right)$holds true. Assume that both $v_{ \pm}$are non-negative. Then by the variational definition of $\xi_{0}$, we have $\tilde{\lambda}\left(v_{+}, v_{-}\right) \geq 0$ and $\tilde{\lambda}(0,0)=0$. For our purposes, it is important to point out that $\tilde{\lambda}\left(v_{+}, v_{-}\right)$is positive whenever at least one of the arguments is. Indeed, if $\tilde{\lambda}\left(v_{+}, v_{-}\right)=0$ and $v_{+}>0$ or $v_{-}>0$, then we deduce from (2.14) that the minimum is achieved by the eigenfunction $\phi_{0}$ of $-\Delta_{\alpha}^{\mathbb{R}}$ corresponding to $\xi_{0}$ and that $\phi_{0}(a)=0$ or $\phi_{0}(-a)=0$. Since $\phi_{0}$ is positive, the latter leads to a contradiction.

If $V_{+}$and $V_{-}$are real-valued functions, then $\tilde{\lambda}$ gives rise to a function $\lambda$ : $\mathbb{R}^{2} \rightarrow \mathbb{R}$ via setting

$$
\lambda(x):=\tilde{\lambda}\left(V_{+}\left(x_{1}\right), V_{-}\left(x_{1}\right)\right) .
$$

It follows from the properties of $\tilde{\lambda}$ that if $V_{ \pm}$are non-negative and $V_{-}$or $V_{+}$is nontrivial (i.e., non-zero on a measurable set of positive Lebesgue measure), then $\lambda$ is a non-trivial non-negative function.

In any case, using Fubini's theorem, we get a fundamental lower bound for our form $\mathcal{E}_{\alpha, V_{+}, V_{-}}$.

Lemma 2.4. For every $\psi \in W^{1,2}$, we have

$$
\mathcal{E}_{\alpha, V_{+}, V_{-}}[\psi]-\xi_{0}\|\psi\|_{L^{2}}^{2} \geq \int_{\mathbb{R}^{2}}\left|\partial_{1} \psi\right|^{2}+\int_{\mathbb{R}^{2}} \lambda|\psi|^{2} .
$$

The above result shows that $H_{\alpha, V_{+}, V_{-}}-\xi_{0}$ is bounded from below by the one-dimensional Schrödinger operator

$$
\left(-\Delta^{\mathbb{R}}+\lambda\right) \otimes 1 \quad \text { on } L^{2}(\mathbb{R}) \otimes L^{2}(\mathbb{R}) .
$$

\section{§2.4. The Krein-like resolvent formula}

The first auxiliary step is to reconstruct the resolvent of the unperturbed Hamiltonian, i.e. $R_{\alpha}(z):=\left(H_{\alpha, 0,0}-z\right)^{-1}$ for $z \in \mathbb{C} \backslash\left[\xi_{0}, \infty\right)$. Once we have $R_{\alpha}(z)$ we 
introduce potentials $V_{ \pm}$and build a Krein-like resolvent of $H_{\alpha, V_{+}, V_{-}}$as a perturbation of $R_{\alpha}(\cdot)$. To do this, we will follow the treatment by Posilicano [22].

2.4.1. The resolvent of the unperturbed Hamiltonian. As above, $\xi_{j}$, with $j \in \mathcal{N}$, stand for the discrete eigenvalues of $-\Delta_{\alpha}^{\mathbb{R}}$, and $\phi_{j}$ denote the corresponding eigenfunctions. Recall that the essential spectrum of $-\Delta_{\alpha}^{\mathbb{R}}$ is purely absolutely continuous. Let $E_{\alpha}^{\mathbb{R}}(\cdot)$ stand for the spectral resolution of $-\Delta_{\alpha}^{\mathbb{R}}$ corresponding to the continuous spectrum and let $P_{\alpha, j}$, with $j \in \mathcal{N}$, denote the eigen-projector $P_{\alpha, j}=$ $\phi_{j}\left(\phi_{j}, \cdot\right)$. Analogously, $E^{\mathbb{R}}(\cdot)$ denotes the spectral resolution of $-\Delta^{\mathbb{R}}$. Using (1.4), one gets

$R_{\alpha}(z)=\sum_{j \in \mathcal{N}} \int_{\mathbb{R}_{+}}\left(\beta+\xi_{j}-z\right)^{-1} \mathrm{~d} E^{\mathbb{R}}(\beta) \otimes P_{\alpha, j}+\int_{\mathbb{R}_{+}^{2}}\left(\beta+\beta^{\prime}-z\right)^{-1} \mathrm{~d} E^{\mathbb{R}}(\beta) \otimes \mathrm{d} E_{\alpha}^{\mathbb{R}}\left(\beta^{\prime}\right)$,

where $z \in \mathbb{C} \backslash\left[\xi_{0}, \infty\right)$. This implies the decomposition

$$
R_{\alpha}(z)=R_{\alpha}^{d}(z)+R_{\alpha}^{c}(z),
$$

where the $R_{\alpha}^{\iota}(z)$, with $\iota \in\{d, c\}$, act on a separated variable function $f(x)=$ $f_{1}\left(x_{1}\right) f_{2}\left(x_{2}\right)$ as

$$
\begin{aligned}
R_{\alpha}^{d}(z) f(x) & =\sum_{j \in \mathcal{N}} \frac{1}{\sqrt{2 \pi}} \int_{\mathbb{R}} \mathrm{d} p_{1} \frac{\widehat{f}_{1}\left(p_{1}\right) \mathrm{e}^{i p_{1} x_{1}}}{p_{1}^{2}+\xi_{j}-z} \phi_{j}\left(x_{2}\right)\left(\phi_{j}, f_{2}\right)_{L^{2}(\mathbb{R})}, \\
R_{\alpha}^{c}(z) f & =\int_{\mathbb{R}_{+}^{2}} \frac{1}{\beta+\beta^{\prime}-z} \mathrm{~d} E^{\mathbb{R}}(\beta) f_{1} \otimes \mathrm{d} E_{\alpha}^{\mathbb{R}}\left(\beta^{\prime}\right) f_{2},
\end{aligned}
$$

with $\widehat{f_{1}}$ denoting the Fourier transform of $f_{1}$. Set $\tau_{j}^{2}(z):=z-\xi_{j}$. In the following we assume that $z$ is taken from the first sheet of the domain of $z \mapsto \tau_{j}(z)$, i.e. $\Im \tau_{j}(z)>0$.

Using the standard representation of the Green function of the one-dimensional Laplace operator,

$$
\left(-\Delta^{\mathbb{R}}-k^{2}\right)^{-1}\left(x_{1}, y_{1}\right)=\frac{1}{2 \pi} \int_{\mathbb{R}} \frac{\mathrm{e}^{i p\left(x_{1}-y_{1}\right)}}{p^{2}-k^{2}} \mathrm{~d} p=\frac{i}{2} \frac{\mathrm{e}^{i k\left|x_{1}-y_{1}\right|}}{k},
$$

we conclude that $R_{\alpha}^{d}(z):=\sum_{j \in \mathcal{N}} R_{\alpha}^{j, d}(z)$ where the $R_{\alpha}^{j, d}(z)$ are integral operators with kernels

$$
G_{\alpha}^{j, d}(z ; x, y)=\frac{i}{2} \frac{\mathrm{e}^{i \tau_{j}(z)\left|x_{1}-y_{1}\right|}}{\tau_{j}(z)} \phi_{j}\left(x_{2}\right) \overline{\phi_{j}\left(y_{2}\right)} .
$$

Moreover

$$
R_{\alpha}^{c}(z) f=\frac{i}{2} \int_{\mathbb{R}^{2}} \int_{\mathbb{R}} \frac{\mathrm{e}^{i \tau(p, z)\left|x_{1}-y_{1}\right|}}{\tau(p, z)} \psi\left(p, x_{2}\right) \overline{\psi\left(p, y_{2}\right)} f_{1}\left(y_{1}\right) f_{2}\left(y_{2}\right) \mathrm{d} y_{1} \mathrm{~d} y_{2} \mathrm{~d} p
$$


where $\tau^{2}(p, z)=z-p^{2}, \Im \tau(p, z)>0$ and $\psi\left(p, x_{2}\right)$ stands for the generalized eigenfunction of $-\Delta_{\alpha}^{\mathbb{R}}$ discussed in [2, Chap. II.2.4]. To be fully specific, $\psi\left(p, x_{2}\right)$ can be obtained from eq. (2.4.1) of [2, Chap. II] multiplying it by $\frac{1}{2 \pi}$; see also [2, Appendix E, eq. (E.5)].

The resolvent of $H_{\alpha, 0,0}$ can also be written in a Krein-like form. We start with the resolvent of the free system $R_{0}(z)=\left(H_{0,0,0}-z\right)^{-1}, z \in \mathbb{C} \backslash[0, \infty)$. By means of the embeddings $I_{ \pm}: W^{1,2} \hookrightarrow L_{ \pm}^{2}$ and their adjoints $I_{ \pm}^{*}: L_{ \pm}^{2} \hookrightarrow W^{-1,2}$, we define

$$
\hat{R}_{0, \pm}(z):=I_{ \pm} R_{0}(z): L^{2} \rightarrow L_{ \pm}^{2}, \quad \check{R}_{0, \pm}(z):=R_{0}(z) I_{ \pm}^{*}: L_{ \pm}^{2} \rightarrow L^{2} .
$$

Finally, the "bilateral" embeddings take the forms

$$
\mathrm{R}_{0, i j}(z):=I_{i} R_{0}(z) I_{j}^{*}: L^{2}(\mathbb{R} \times\{j a\}) \rightarrow L^{2}(\mathbb{R} \times\{i a\}), \quad i, j \in\{+,-\} .
$$

Let $\Gamma_{0, \alpha}(z)$ denote the operator-valued matrix acting in $L_{+}^{2} \oplus L_{-}^{2}$ as

$$
\Gamma_{0, \alpha}(z):=\left(\begin{array}{cc}
-\alpha^{-1} & 0 \\
0 & -\alpha^{-1}
\end{array}\right)+\left(\begin{array}{ll}
\mathrm{R}_{0,++}(z) & \mathrm{R}_{0,+-}(z) \\
\mathrm{R}_{0,-+}(z) & \mathrm{R}_{0,--}(z)
\end{array}\right) .
$$

Note that the continuity of $I_{i}: W^{1,2} \hookrightarrow L_{i}^{2}, i \in\{+,-\}$, implies the continuity of the adjoint embedding $I_{i}^{*}: L_{i}^{2} \hookrightarrow W^{-1,2}$. Therefore

$$
\operatorname{Ran} \check{R}_{0, i}(z) \subset W^{1,2} \text {. }
$$

Moreover, since $\operatorname{Ran} I_{i}^{*} \cap L^{2}=\{0\}$, we obtain

$$
\operatorname{Ran} \check{R}_{0, i}(z) \cap W^{2,2}=\{0\} .
$$

Theorem 2.5. Let $z \in \mathbb{C} \backslash\left[\xi_{0}, \infty\right)$ be such that the operator $\Gamma_{0, \alpha}(z)$ is invertible with bounded inverse. Then the resolvent $R_{\alpha}(z)$ is given by

$$
R_{\alpha}(z)=R_{0}(z)-\sum_{i, j \in\{+,-\}} \check{R}_{0, i}(z) \Gamma_{0, \alpha}(z)_{i j}^{-1} \hat{R}_{0, j}(z),
$$

where the $\Gamma_{0, \alpha}(z)_{i j}^{-1}$ are the matrix elements of the inverse of $\Gamma_{0, \alpha}(z)$.

Proof. In view of (2.19), the operator $R_{\alpha}(z)$ defined by (2.21) satisfies $\operatorname{Ran} R_{\alpha}(z)$ $\subset W^{1,2}$. Assume $\psi \in W^{1,2}$ and $\phi \in L^{2}$. Using $\mathcal{E}_{0,0,0}\left(\psi, R_{0}(z) \phi\right)-z\left(\psi, R_{0}(z) \phi\right)=$ $(\psi, \phi)$, we get

$$
\begin{aligned}
\mathcal{E}_{\alpha, 0,0}\left(\psi, R_{\alpha}(z) \phi\right) & -z\left(\psi, R_{\alpha}(z) \phi\right)=(\psi, \phi) \\
& -\sum_{i \in\{+,-\}} \alpha\left(\psi, \hat{R}_{0, i}(z) \phi\right)_{L_{i}^{2}}-\sum_{i, j \in\{+,-\}}\left(\psi, \Gamma_{0, \alpha}(z)_{i j}^{-1} \hat{R}_{0, j}(z) \phi\right)_{L_{i}^{2}} \\
& +\sum_{i, j, k \in\{+,-\}} \alpha\left(\psi, \mathrm{R}_{0, i j}(z) \Gamma_{0, \alpha}(z)_{j k}^{-1} \hat{R}_{0, k}(z) \phi\right)_{L_{i}^{2}},
\end{aligned}
$$


where we use the embedding $W^{1,2} \hookrightarrow L_{i}^{2}$ for $\psi$ in all expressions with scalar product in $L_{i}^{2}$. Applying the identity

$$
\left(\psi, \hat{R}_{0, i}(z) \phi\right)_{L_{i}^{2}}=\sum_{j, k \in\{+,-\}}\left(\psi, \Gamma_{0, \alpha}(z)_{i k} \Gamma_{0, \alpha}(z)_{k j}^{-1} \hat{R}_{0, j}(z) \phi\right)_{L_{i}^{2}}
$$

and using the explicit form of $\Gamma_{0, \alpha}(z)_{i j}$, one obtains

$$
\mathcal{E}_{\alpha, 0,0}\left(\psi, R_{\alpha}(z) \phi\right)-z\left(\psi, R_{\alpha}(z) \phi\right)=(\psi, \phi) .
$$

Moreover, note that $R_{\alpha}(z)$ is invertible. Indeed, employing (2.21) and (2.20) we conclude that each $f \in \operatorname{Ran} R_{\alpha}(z)$ is a direct sum $f=f_{1} \dot{+} f_{2}$ where $f_{1} \in$ Ran $R_{0}(z)$ and $f_{2} \in W^{1,2} \backslash W^{2,2}$. This implies Ker $R_{\alpha}=\{0\}$ and together with (2.22) completes the proof.

2.4.2. The Krein-like formula for the resolvent of $H_{\alpha, V_{+}, V_{-}}$. Relying on (2.21) and (2.19), we can determine analogous embedding operators as in the previous section but now instead of $R_{0}(z)$ we consider $R_{\alpha}(z)$. Namely, define $\hat{R}_{\alpha, \pm}(z):=I_{ \pm} R_{\alpha}(z): L^{2} \rightarrow L_{ \pm}^{2}, \check{R}_{\alpha, \pm}(z):=R_{\alpha}(z) I_{ \pm}^{*}: L_{ \pm}^{2} \rightarrow L^{2}$ and $\mathrm{R}_{\alpha, i j}(z):=$ $I_{i} R_{\alpha}(z) I_{j}^{*}: L_{j}^{2} \rightarrow L_{i}^{2}$ where $i, j \in\{+,-\}$.

For any bounded function $V$, set $V^{1 / 2}:=\operatorname{sgn}(V)|V|^{1 / 2}$. Define the operatorvalued matrix

$$
B(z):=\left(\begin{array}{ll}
\left|V_{+}\right|^{1 / 2} \mathrm{R}_{\alpha,++}(z) V_{+}^{1 / 2} & \left|V_{+}\right|^{1 / 2} \mathrm{R}_{\alpha,+-}(z) V_{-}^{1 / 2} \\
\left|V_{-}\right|^{1 / 2} \mathrm{R}_{\alpha,-+}(z) V_{+}^{1 / 2} & \left|V_{-}\right|^{1 / 2} \mathrm{R}_{\alpha,--}(z) V_{-}^{1 / 2}
\end{array}\right)
$$

acting on $L_{+}^{2} \oplus L_{-}^{2}$.

Theorem 2.6. Let $z \in \mathbb{C} \backslash \sigma\left(H_{\alpha, V_{+}, V_{-}}\right)$be such that the operator $1+B(z)$ is invertible with bounded inverse. Then

$$
R_{\alpha, V_{+}, V_{-}}(z)=R_{\alpha}(z)-\sum_{i, j \in\{+,-\}} \check{R}_{\alpha, i}(z) V_{i}^{1 / 2}[1+B(z)]_{i j}^{-1}\left|V_{j}\right|^{1 / 2} \hat{R}_{\alpha, j}(z)
$$

determines the resolvent of $H_{\alpha, V_{+}, V_{-}}$; here $[1+B(z)]_{i j}^{-1}$ stand for the operator valued matrix elements of $[1+B(z)]^{-1}$.

Proof. Combining (2.21) and (2.19) and using the definition of $R_{\alpha, V_{+}, V_{-}}(z)$ given by (2.23) we have $\operatorname{Ran} \breve{R}_{\alpha, i}(z) \subset W^{1,2}$ and consequently $\operatorname{Ran} R_{\alpha, V_{+}, V_{-}}(z) \subset W^{1,2}$.

Denote $\Upsilon(z)_{i j}:=[1+B(z)]_{i j}^{-1}$ and assume $\psi \in W^{1,2}$ and $\phi \in L^{2}$. Employing the identity $\mathcal{E}_{\alpha, 0,0}\left(\psi, R_{\alpha}(z) \phi\right)-z\left(\psi, R_{\alpha}(z) \phi\right)=(\psi, \phi)$ we get 


$$
\begin{aligned}
\mathcal{E}_{\alpha, V_{+}, V_{-}}\left(\psi, R_{\alpha, V_{+}, V_{-}}(z) \phi\right)-z\left(\psi, R_{\alpha, V_{+}, V_{-}}(z) \phi\right)=(\psi, \phi) & \\
& +\sum_{i \in\{+,-\}}\left(\psi, V_{i} \hat{R}_{\alpha, i}(z) \phi\right)_{L_{i}^{2}}-\sum_{i, j \in\{+,-\}}\left(\psi, V_{i}^{1 / 2} \Upsilon(z)_{i j}\left|V_{j}\right|^{1 / 2} \hat{R}_{\alpha, j}(z) \phi\right)_{L_{i}^{2}} \\
& -\sum_{i, j, k \in\{+,-\}}\left(\psi, V_{i} \mathrm{R}_{\alpha, i j}(z) V_{j}^{1 / 2} \Upsilon(z)_{j k}\left|V_{k}\right|^{1 / 2} \hat{R}_{\alpha, k}(z) \phi\right)_{L_{i}^{2}} .
\end{aligned}
$$

Furthermore, writing

$$
\left(\psi, V_{i} \hat{R}_{\alpha, i}(z) \phi\right)_{L_{i}^{2}}=\sum_{j, k \in\{+,-\}}\left(\psi, V_{i}^{1 / 2} \Upsilon(z)_{i k}^{-1} \Upsilon(z)_{k j}\left|V_{j}\right|^{1 / 2} \hat{R}_{\alpha, j}(z) \phi\right)_{L_{i}^{2}}
$$

and using the explicit form of $\Upsilon(z)_{i j}$, one obtains

$$
\mathcal{E}_{\alpha, V_{+}, V_{-}}\left(\psi, R_{\alpha, V_{+}, V_{-}}(z) \phi\right)-z\left(\psi, R_{\alpha, V_{+}, V_{-}}(z) \phi\right)=(\psi, \phi) .
$$

Repeating the same argument as in the proof Theorem 2.5, we conclude that $R_{\alpha, V_{+}, V_{-}}(z)$ is invertible.

The resolvent formula derived in the above theorem can be written in a short, more familiar form. To this end it is convenient to write $\mathrm{V}^{1 / 2}=V_{+}^{1 / 2} \oplus V_{-}^{1 / 2}$ (and analogously for $\left.|\mathrm{V}|^{1 / 2}\right)$ and introduce $\hat{R}_{\alpha}(z): L^{2} \rightarrow L_{+}^{2} \oplus L_{-}^{2}$ defined by $\hat{R}_{\alpha}(z) \psi=$ $\hat{R}_{\alpha,+}(z) \psi \oplus \hat{R}_{\alpha,-}(z) \psi$ and $\check{R}_{\alpha}(z): L_{+}^{2} \oplus L_{-}^{2} \rightarrow L^{2}$ defined by $\check{R}_{\alpha}(z)\left(f_{+} \oplus f_{-}\right)=$ $\check{R}_{\alpha,+}(z) f_{+}+\check{R}_{\alpha,-}(z) f_{-}$. With these notations, identity (2.23) reads

$$
R_{\alpha, V_{+}, V_{-}}(z)=R_{\alpha}(z)-\check{R}_{\alpha}(z) \mathrm{V}^{1 / 2}[1+B(z)]^{-1}|\mathrm{~V}|^{1 / 2} \hat{R}_{\alpha}(z) .
$$

Note that (2.24) gives the Krein-like resolvent studied by Posilicano [22, 23]. (To be fully specific we identify the embedding $\eta$ introduced in [22] with $W^{1,2} \hookrightarrow$ $L^{2}\left(\mathbb{R} \times\{+a\},\left|V_{+}\right|^{1 / 2} d x\right) \oplus L^{2}\left(\mathbb{R} \times\{-a\},\left|V_{-}\right|^{1 / 2} d x\right)$.) Furthermore, redefining all the embeddings introduced above by means of $\eta$, we get the resolvent formula derived in [22, Thm. 2.1]. Applying the results of [23], we get

$$
z \in \rho\left(H_{\alpha}\right) \cap \rho\left(H_{\alpha, V_{+}, V_{-}}\right) \Leftrightarrow z \in \rho(1+B(z))
$$

and

$$
z \in \sigma_{\mathrm{p}}\left(H_{\alpha, V_{+}, V_{-}}\right) \Leftrightarrow \operatorname{Ker}(1+B(z)) \neq\{0\} .
$$

2.4.3. The system with mirror symmetry. Let us consider a system with mirror symmetry, i.e.

$$
V_{+}=V_{0}=V_{-},
$$

where $V_{0}: \mathbb{R} \rightarrow \mathbb{R}$ is a given function. For this case we use a special notation, $H_{\alpha, V_{0}}:=H_{\alpha, V_{0}, V_{0}}$. Using (2.23), we can reconstruct the resolvent $R_{\alpha, V_{0}}(z):=$ $\left(H_{\alpha, V_{0}}-z\right)^{-1}$. 
Now, we introduce a "slight" perturbation of the symmetry taking

$$
\begin{aligned}
& V_{+}=V+V_{\epsilon}, \quad \text { where } \quad V_{\epsilon}:=\epsilon V_{p}, \quad \epsilon>0, \\
& V_{-}=V,
\end{aligned}
$$

where the "perturbant" $V_{p}$ is a function from $L^{\infty}(\mathbb{R})$. In the following we abbreviate $H_{\alpha ; \epsilon}:=H_{\alpha, V+V_{\epsilon}, V}$.

Considering the system governed by $H_{\alpha, V_{0}}$ as a starting point, we construct the resolvent $R_{\alpha ; \epsilon}$ of $H_{\alpha ; \epsilon}$,

$$
R_{\alpha ; \epsilon}(z)=R_{\alpha, V_{0}}(z)-\check{R}_{\alpha,+}(z) V_{\epsilon}^{1 / 2} \Gamma(z)^{-1}\left|V_{\epsilon}\right|^{1 / 2} \hat{R}_{\alpha,+}(z)
$$

where

$$
\Gamma(z):=1+\left|V_{\epsilon}\right|^{1 / 2} \mathrm{R}_{\alpha, V_{0}}(z) V_{\epsilon}^{1 / 2}
$$

and $\mathrm{R}_{\alpha, V_{0}}$ acts as $R_{\alpha, V_{0}, V_{0}}$ but maps $L_{+}^{2}$ to $L_{+}^{2}$. The proof of (2.28) can be obtained by repeating the arguments of the proof of Theorem 2.6.

\section{§3. The essential spectrum}

The spectrum of the unperturbed Hamiltonian $H_{\alpha, 0,0}$ is given by (1.4). In the following we show that the essential spectrum (1.5) is stable under perturbations $V_{ \pm}$ which vanish at infinity in the following sense:

$$
\lim _{L \rightarrow \infty} \operatorname{ess} \sup _{\mathbb{R} \backslash[-L, L]}\left|V_{ \pm}\right|=0 .
$$

Theorem 3.1 (Essential spectrum). Assume (3.30) holds. Then

$$
\sigma_{\text {ess }}\left(H_{\alpha, V_{+}, V_{-}}\right)=\left[\xi_{0}, \infty\right) .
$$

We prove this theorem in two steps.

\section{§3.1. A lower bound for the essential spectrum threshold}

First, we show that the threshold of the essential spectrum does not descend below the energy $\xi_{0}$.

Lemma 3.2. Assume (3.30) holds. Then

$$
\inf \sigma_{\text {ess }}\left(H_{\alpha, V_{+}, V_{-}}\right) \geq \xi_{0} .
$$

Proof. Given a number $L>a$, let $H^{N}$ denote the operator $H_{\alpha, V_{+}, V_{-}}$with a supplementary Neumann condition imposed on the lines $\{ \pm L\} \times \mathbb{R}$ and segments $(-L, L) \times\{ \pm L\}$. It is conventionally introduced as the operator associated with the quadratic form $\mathcal{E}^{N}$ which acts in the same way as $\mathcal{E}_{\alpha, V_{+}, V_{-}}$but has a larger 
domain, $\mathfrak{D}\left(\mathcal{E}^{N}\right):=\bigoplus_{k=0}^{4} W^{1,2}\left(\Omega_{k}\right)$, where $\Omega_{k}$ are the connected components of $\mathbb{R}^{2}$ divided by the curves where the Neumann condition is imposed:

$$
\begin{array}{lll}
\Omega_{0}:=(-L, L)^{2}, & \Omega_{1}:=(-L, L) \times(-\infty,-L), & \Omega_{3}:=(-\infty,-L) \times \mathbb{R}, \\
& \Omega_{2}:=(-L, L) \times(L, \infty), & \Omega_{4}:=(L, \infty) \times \mathbb{R} .
\end{array}
$$

We have the decomposition $H^{N}=\bigoplus_{k=0}^{4} H_{k}^{N}$, where $H_{k}^{N}$ are the operators associated on $L^{2}\left(\Omega_{k}\right)$ with the quadratic forms

$$
\begin{aligned}
\mathcal{E}_{k}^{N}[\psi]:= & \int_{\Omega_{k}}|\nabla \psi|^{2}+\int_{(\mathbb{R} \times\{+a\}) \cap \Omega_{k}}\left(V_{+}-\alpha\right)\left|I_{+} \psi\right|^{2} \\
& +\int_{(\mathbb{R} \times\{-a\}) \cap \Omega_{k}}\left(V_{-}-\alpha\right)\left|I_{-} \psi\right|^{2}, \\
\mathfrak{D}\left(\mathcal{E}_{k}^{N}\right):= & W^{1,2}\left(\Omega_{k}\right) .
\end{aligned}
$$

Since $H_{\alpha, V_{+}, V_{-}} \geq H^{N}$ and the spectrum of $H_{0}^{N}$ is purely discrete, the minimax principle gives the estimate

$$
\inf \sigma_{\mathrm{ess}}\left(H_{\alpha, V_{+}, V_{-}}\right) \geq \min _{k \in\{1, \ldots, 4\}}\left\{\inf \sigma_{\mathrm{ess}}\left(H_{k}^{N}\right)\right\} \geq \min _{k \in\{1, \ldots, 4\}}\left\{\inf \sigma\left(H_{k}^{N}\right)\right\} .
$$

It is easy to see that the spectra of $H_{1}^{N}$ and $H_{2}^{N}$ coincide with the non-negative semi-axis $[0, \infty)$. Hence, it remains to analyze the bottoms of the spectra of $H_{3}^{N}$ and $H_{4}^{N}$. Using analogous arguments to those leading to Lemma 2.4, we have the lower bound $(k \in\{3,4\})$

$$
\mathcal{E}_{k}^{N}[\psi]-\xi_{0}\|\psi\|_{L^{2}\left(\Omega_{k}\right)}^{2} \geq \int_{\Omega_{k}}\left|\partial_{1} \psi\right|^{2}+\int_{\Omega_{k}} \lambda|\psi|^{2} \geq \underset{\Omega_{k}}{\operatorname{essinf}} \lambda\|\psi\|_{L^{2}\left(\Omega_{k}\right)}^{2} .
$$

Consequently,

$$
\inf \sigma_{\mathrm{ess}}\left(H_{\alpha, V_{+}, V_{-}}\right) \geq \min \left\{0, \xi_{0}+\min \left\{\underset{\Omega_{3}}{\operatorname{essinf}} \lambda, \underset{\Omega_{4}}{\operatorname{essinf}} \lambda\right\}\right\} .
$$

Now, since $V_{ \pm}$vanish at infinity, the same is true for the function $\lambda$. Hence, for every $\epsilon$, there exists $L$ such that for a.e. $\left|x_{1}\right|>L\left(x_{2} \in \mathbb{R}\right)$, we have $|\lambda(x)|<\epsilon$. Consequently, if $\epsilon<\left|\xi_{0}\right|$,

$$
\inf \sigma_{\text {ess }}\left(H_{\alpha, V_{+}, V_{-}}\right) \geq \xi_{0}-\epsilon
$$

The claim then follows by the fact that $\epsilon$ can be chosen arbitrarily small.

\section{§3.2. The opposite inclusion}

Lemma 3.3. Assume (3.30) holds. Then

$$
\sigma_{\text {ess }}\left(H_{\alpha, V_{+}, V_{-}}\right) \supseteq\left[\xi_{0}, \infty\right) .
$$

Proof. Our proof is based on the Weyl criterion adapted to quadratic forms in [7] and applied to quantum waveguides in [21]. By this general characterization of 
essential spectrum and since the set $\left[\xi_{0}, \infty\right)$ has no isolated points, it is enough to find for every $\xi \in\left[\xi_{0}, \infty\right)$ a sequence $\left\{\psi_{n}\right\}_{n=1}^{\infty} \subseteq \mathfrak{D}\left(\mathcal{E}_{\alpha, V_{+}, V_{-}}\right)$such that

(i) $\forall n \in \mathbb{N} \backslash\{0\},\left\|\psi_{n}\right\|_{L^{2}}=1$,

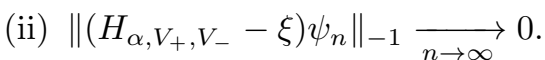

The symbol $\|\cdot\|_{-1}$ denotes the norm in $\mathfrak{D}\left(\mathcal{E}_{\alpha, V_{+}, V_{-}}\right)^{*}$, the dual of $\mathfrak{D}\left(\mathcal{E}_{\alpha, V_{+}, V_{-}}\right)$ equipped with the norm

$$
\|\psi\|_{+1}:=\sqrt{\mathcal{E}_{\alpha, V_{+}, V_{-}}[\psi]+\left(1+C_{0}\right)\|\psi\|_{L^{2}}^{2}},
$$

where $C_{0}$ denotes any positive constant such that $H_{\alpha, V_{+}, V_{-}}+C_{0}$ is a non-negative operator. We choose the constant $C_{0}$ sufficiently large, so that $\|\cdot\|_{+1}$ is equivalent to the usual norm in $W^{1,2}$. This is possible in view of the boundedness of $V_{ \pm}$and Lemma 2.1.

Let $n \in \mathbb{N} \backslash\{0\}$. Given $k \in \mathbb{R}$, we set $\xi:=\xi_{0}+k^{2}$. Recall that the function $\phi_{0}$ denotes the ground state of $-\Delta_{\alpha}^{\mathbb{R}}$ corresponding to $\xi_{0}$. Since the interactions $V_{ \pm}$ vanish at infinity, good candidates for the sequence $\psi_{n}$ seem to be plane waves in the $x_{1}$-direction "localized at infinity" and modulated by $\phi_{0}$ in the $x_{2}$-direction. More precisely,

$$
\psi_{n}(x):=\varphi_{n}\left(x_{1}\right) \phi_{0}\left(x_{2}\right) e^{i k x_{1}},
$$

where $\varphi_{n}\left(x_{1}\right):=n^{-1 / 2} \varphi\left(x_{1} / n-n\right)$ with $\varphi$ being a non-zero $C^{\infty}$ function with compact support in $(-1,1)$. Note that $\operatorname{supp} \varphi_{n} \subset\left(n^{2}-n, n^{2}+n\right)$. Clearly, $\psi_{n} \in$ $W^{1,2}=\mathfrak{D}\left(\mathcal{E}_{\alpha, V_{+}, V_{-}}\right)$. To satisfy (i), we assume that both $\phi_{0}$ and $\varphi$ have $L^{2}(\mathbb{R})$ norm 1. It remains to verify condition (ii).

By the definition of the dual norm, we have

$$
\left\|\left(H_{\alpha, V_{+}, V_{-}}-\xi\right) \psi_{n}\right\|_{-1}=\sup _{\eta \in W^{1,2} \backslash\{0\}} \frac{\left|\mathcal{E}_{\alpha, V_{+}, V_{-}}\left(\eta, \psi_{n}\right)-\xi\left(\eta, \psi_{n}\right)_{L^{2}}\right|}{\|\eta\|_{+1}} .
$$

An explicit computation using integration by parts yields

$$
\begin{aligned}
\mathcal{E}_{\alpha, V_{+}, V_{-}}\left(\eta, \psi_{n}\right)-\xi\left(\eta, \psi_{n}\right)_{L^{2}} & \\
& =\left(\eta,\left[-\ddot{\varphi}_{n}-2 i k \dot{\varphi}_{n}\right] \phi_{0} e^{i k x_{1}}\right)_{L^{2}}+\int_{\mathbb{R} \times\{a\}} V_{+} I_{+}\left(\bar{\eta} \psi_{n}\right)+\int_{\mathbb{R} \times\{-a\}} V_{-} I_{-}\left(\bar{\eta} \psi_{n}\right) .
\end{aligned}
$$

Using the Schwarz inequality and the normalization of $\phi_{0}$, we get the estimates

$$
\begin{aligned}
\left|\left(\eta,\left[-\ddot{\varphi}_{n}-2 i k \dot{\varphi}_{n}\right] \phi_{0} e^{i k x_{1}}\right)_{L^{2}}\right| & \leq\|\eta\|_{L^{2}}\left(\left\|\ddot{\varphi}_{n}\right\|_{L^{2}(\mathbb{R})}+2|k|\left\|\dot{\varphi}_{n}\right\|_{L^{2}(\mathbb{R})}\right), \\
\left|\int_{\mathbb{R} \times\{ \pm a\}} V_{ \pm} I_{ \pm}\left(\bar{\eta} \psi_{n}\right)\right| & \leq\left\|I_{ \pm} \eta\right\|_{L_{ \pm}^{2}}\left\|V_{ \pm} \varphi_{n}\right\|_{L^{2}(\mathbb{R})}\left|\phi_{0}( \pm a)\right| .
\end{aligned}
$$


By the choice of $C_{0}$ and Lemma 2.1, both $\|\eta\|_{L^{2}}$ and $\left\|I_{ \pm} \eta\right\|_{L_{ \pm}^{2}}$ can be bounded by a constant times $\|\eta\|_{+1}$. Hence, there is a constant $C$, depending on $a, \alpha, k$, $\left\|V_{+}\right\|_{\infty}$ and $\left\|V_{-}\right\|_{\infty}$, such that

$$
\begin{aligned}
\|\left(H_{\alpha, V_{+}, V_{-}}\right. & -\xi) \psi_{n} \|_{-1} \\
& \leq C\left(\left\|\ddot{\varphi}_{n}\right\|_{L^{2}(\mathbb{R})}+\left\|\dot{\varphi}_{n}\right\|_{L^{2}(\mathbb{R})}+\left\|V_{+} \varphi_{n}\right\|_{L^{2}(\mathbb{R})}+\left\|V_{-} \varphi_{n}\right\|_{L^{2}(\mathbb{R})}\right)
\end{aligned}
$$

The first two norms on the right hand side tend to zero as $n \rightarrow \infty$ because

$$
\left\|\dot{\varphi}_{n}\right\|_{L^{2}(\mathbb{R})}=n^{-1}\|\dot{\varphi}\|_{L^{2}(\mathbb{R})}, \quad\left\|\ddot{\varphi}_{n}\right\|_{L^{2}(\mathbb{R})}=n^{-2}\|\ddot{\varphi}\|_{L^{2}(\mathbb{R})} .
$$

The remaining terms tend to zero because of the estimate

$$
\left\|V_{ \pm} \varphi_{n}\right\|_{L^{2}(\mathbb{R})} \leq \operatorname{ess} \sup _{\operatorname{supp} \varphi_{n}}\left|V_{ \pm}\right|
$$

in which we have employed the normalization of $\varphi_{n}$, and the fact that $\inf \operatorname{supp} \varphi_{n}$ tends to infinity as $n \rightarrow \infty$.

\section{$\S 4$. The point spectrum}

In this section we study the existence of eigenvalues corresponding to bound states. We will be interested in the discrete spectrum as well as in embedded eigenvalues.

\section{§4.1. The discrete spectrum}

In the following we establish a condition which guarantees the existence of discrete eigenvalues outside the essential spectrum.

Theorem 4.1 (Discrete spectrum). Assume that $V_{-}+V_{+} \in L^{1}(\mathbb{R})$ and

$$
\int_{\mathbb{R}}\left(V_{-}+V_{+}\right)(x) \mathrm{d} x<0
$$

Then

$$
\inf \sigma\left(H_{\alpha, V_{+}, V_{-}}\right)<\xi_{0} .
$$

Consequently, if in addition $V_{ \pm}$vanish at infinity, then $H_{\alpha, V_{+}, V_{-}}$has an isolated eigenvalue of finite multiplicity below $\xi_{0}$, i.e. (1.7) holds.

Proof. The proof is based on a variational argument. Our aim is to find a test function $\psi \in W^{1,2}$ such that

$$
Q[\psi]:=\mathcal{E}_{\alpha, V_{+}, V_{-}}[\psi]-\xi_{0}\|\psi\|_{L^{2}\left(\mathbb{R}^{2}\right)}^{2}<0 .
$$

For any $n \in \mathbb{N} \backslash\{0\}$, we set

$$
\psi_{n}(x):=\varphi_{n}\left(x_{1}\right) \phi_{0}\left(x_{2}\right),
$$


where $\phi_{0}$ is, as before, a positive eigenfunction of $-\Delta_{\alpha}^{\mathbb{R}}$, normalized to 1 in $L^{2}(\mathbb{R})$, and

$$
\varphi_{n}\left(x_{1}\right):= \begin{cases}1 & \text { if }\left|x_{1}\right| \leq n, \\ \frac{2 n-\left|x_{1}\right|}{n} & \text { if } n \leq\left|x_{1}\right| \leq 2 n, \\ 0 & \text { otherwise. }\end{cases}
$$

Obviously, $\psi_{n} \in W^{1,2}$. Using $\varepsilon_{\alpha}\left[\phi_{0}\right]=\xi_{0}\left\|\phi_{0}\right\|_{L^{2}(\mathbb{R})}^{2}$ and the fact that $\phi_{0}$ is even, it is easy to check the identity

$$
Q\left[\psi_{n}\right]=\int_{\mathbb{R}}\left|\varphi_{n}^{\prime}\right|^{2}+\left|\phi_{0}(a)\right|^{2} \int_{\mathbb{R}}\left(V_{-}+V_{+}\right)\left|\varphi_{n}\right|^{2} .
$$

As $n \rightarrow \infty$, the first term on the right hand side tends to zero, while the second converges (by the dominated convergence theorem) to a multiple of $\int_{\mathbb{R}}\left(V_{-}+V_{+}\right)<0$. Hence, $Q\left[\psi_{n}\right]$ is negative for $n$ sufficiently large.

Remark 4.2. The integrability of $V_{+}+V_{-}$is just a technical assumption in Theorem 4.1. It is clear from the proof that it is only important to ensure that the quantity

$$
\int_{\mathbb{R}}\left(V_{-}+V_{+}\right)\left|\varphi_{n}\right|^{2}
$$

becomes negative as $n \rightarrow \infty$, the value $-\infty$ for the limit being admissible in principle. For instance, one can alternatively assume that $V_{+}+V_{-}$is non-trivial and non-positive on $\Omega_{0}$ for the present proof to work.

\section{$\S 4.2$. Embedded eigenvalues}

The aim of this section is to show that the system with mirror symmetry (cf. Section 2.4.3) has embedded eigenvalues under certain assumptions. For $V_{0} \in L^{\infty}(\mathbb{R})$ vanishing at infinity, we consider the Hamiltonian $H_{\alpha, V_{0}} \equiv H_{\alpha, V_{0}, V_{0}}$. Recall that the essential spectrum of $H_{\alpha, V_{0}}$ is given by (1.6).

We simultaneously consider an auxiliary operator $H_{\alpha, V_{0}}^{D}$ on $L^{2}\left(\mathbb{R} \times \mathbb{R}^{+}\right)$, which acts as $H_{\alpha, V_{0}}$ on $\mathbb{R} \times \mathbb{R}^{+}$, subject to Dirichlet boundary conditions on $\mathbb{R} \times\{0\}$. It is introduced as the operator on $L^{2}\left(\mathbb{R} \times \mathbb{R}^{+}\right)$associated with the form

$$
\begin{aligned}
\mathcal{E}_{\alpha, V_{0}}^{D}[\psi] & :=\int_{\mathbb{R} \times \mathbb{R}^{+}}|\nabla \psi|^{2}+\int_{\mathbb{R} \times\{a\}}\left(V_{0}-\alpha\right)\left|I_{+} \psi\right|^{2}, \\
\mathfrak{D}\left(\mathcal{E}_{\alpha, V_{0}}^{D}\right) & :=W_{0}^{1,2}\left(\mathbb{R} \times \mathbb{R}^{+}\right),
\end{aligned}
$$

where we keep the same notation $I_{+}$for the embedding $I_{+}: W_{0}^{1,2}\left(\mathbb{R} \times \mathbb{R}^{+}\right) \hookrightarrow L_{+}^{2}$. Since $V_{0}$ vanishes at infinity, it can be shown in the same way as in Section 3 that

$$
\sigma_{\text {ess }}\left(H_{\alpha, V}^{D}\right)=\left[\mu_{0}, \infty\right),
$$


where $\mu_{0} \in\left(\xi_{0}, 0\right]$ is the spectral threshold of the one-dimensional operator $h_{\alpha}^{D}$ on $L^{2}\left(\mathbb{R}_{+}\right)$associated with the form

$$
\varepsilon_{\alpha}^{D}[\phi]:=\int_{\mathbb{R}_{+}}\left|\phi^{\prime}\right|^{2}-\alpha|\phi(a)|^{2}, \quad \mathfrak{D}\left(\varepsilon_{\alpha}^{D}\right):=W_{0}^{1,2}\left(\mathbb{R}_{+}\right) .
$$

Proposition 4.3. One has $\sigma_{\mathrm{ess}}\left(h_{\alpha}^{D}\right)=[0, \infty)$ and

$$
\sigma_{\text {disc }}\left(h_{\alpha}^{D}\right)= \begin{cases}\left\{\xi_{1}\right\} & \text { if } \alpha a>1 \\ \emptyset & \text { if } \alpha a \leq 1\end{cases}
$$

Consequently,

$$
\mu_{0}= \begin{cases}\xi_{1} & \text { if } \alpha a>1 \\ 0 & \text { if } \alpha a \leq 1\end{cases}
$$

Proof. By the methods of Section 3, it is easy to see that the essential spectrum of $h_{\alpha}^{D}$ is the non-negative semi-axis. Note that the existence of a negative eigenvalue of $h_{\alpha}^{D}$ is equivalent to the existence of an eigenfunction of $-\Delta_{\alpha}^{\mathbb{R}}$ corresponding to a negative eigenvalue and vanishing on $\mathbb{R} \times\{0\}$. The latter holds if, and only if, the operator $-\Delta_{\alpha}^{\mathbb{R}}$ has an odd eigenfunction $\phi_{1}$, i.e. $\alpha a>1$. This means that $h_{\alpha}^{D}$ has a negative eigenvalue which is $\xi_{1}$ if, and only if, $\alpha a>1$; otherwise $\sigma_{\text {disc }}\left(h_{\alpha}^{D}\right)=\emptyset$.

Theorem 4.4 (Embedded eigenvalues). Let $V_{0}$ vanish at infinity. Assume that $H_{\alpha, V_{0}}^{D}$ has a (discrete) eigenvalue $\nu$ below $\mu_{0}$. Then $\nu$ is an eigenvalue of $H_{\alpha, V_{0}}$. More specifically, if $\nu \geq \xi_{0}$ (respectively, $\nu<\xi_{0}$ ), then $\nu$ is an embedded (respectively, discrete) eigenvalue of $H_{\alpha, V_{0}}$.

Proof. Let $\psi$ be an eigenfunction of $H_{\alpha, V_{0}}^{D}$ corresponding to $\nu$. By mirror symmetry, the odd extension of $\psi$ to $\mathbb{R}^{2}$ is an eigenfunction of $H_{\alpha, V_{0}}$ corresponding to the same value $\nu$. The rest follows from the fact that the essential spectrum of $H_{\alpha, V_{0}}^{D}$ is strictly contained in the essential spectrum of $H_{\alpha, V_{0}}$.

The following result makes Theorem 4.4 non-void.

Proposition 4.5. Assume $\alpha a>1$. Suppose $V_{0} \in L^{1}(\mathbb{R})$ vanishes at infinity and $\int_{\mathbb{R}} V_{0}(x) \mathrm{d} x<0$. Then $\sigma_{\text {disc }}\left(H_{\alpha, V_{0}}^{D}\right) \neq \emptyset$.

Proof. It follows from Proposition 4.3 that, under the condition $\alpha a>1, h_{\alpha}^{D}$ has a negative eigenvalue $\xi_{1}$ with eigenfunction $\phi_{1}$ (restricted to $\mathbb{R} \times \mathbb{R}_{+}$). Then the claim follows by using the test function $\psi(x):=\varphi_{n}\left(x_{1}\right) \phi_{1}\left(x_{2}\right)$, where $\varphi_{n}$ is introduced in (4.31), as in the proof of Theorem 4.1. 
Finally, to ensure the existence of embedded eigenvalues by Theorem 4.4, it remains to verify that the discrete eigenvalue $\nu$ of $H_{\alpha, V_{0}}^{D}$ (which exists under the hypotheses of Proposition 4.5) can be made larger than or equal to $\xi_{0}$. However, this happens, for instance, in the weak-coupling regime.

Corollary 4.6. Assume a $\alpha>1$. Suppose $V_{0} \in L^{1}(\mathbb{R})$ vanishes at infinity and $\int_{\mathbb{R}} V_{0}(x) \mathrm{d} x<0$. Then there exists $\varepsilon>0$ such that $H_{\alpha, \varepsilon V_{0}}$ has an embedded eigenvalue in $\left[\xi_{0}, \mu_{0}\right)$.

\section{$\S 5$. Hardy inequalities}

In this section we study the case when $V_{+}$and $V_{-}$are non-negative. It is easy to see that in this situation the spectrum does not start below $\xi_{0}$. The purpose of this subsection is to show that a stronger result holds in the present setting. We derive a functional, Hardy-type inequality for $H_{\alpha, V_{+}, V_{-}}$with non-trivial non-negative $V_{+}$ and $V_{-}$, quantifying the repulsive character of the line interactions in this case.

The Hardy-type inequality follows immediately from Lemma 2.4. Indeed, neglecting the kinetic term in (2.16), we arrive at

Theorem 5.1 (Local Hardy inequality). Assume that $V_{ \pm}$are non-negative and that $V_{-}$or $V_{+}$is non-trivial. Then

$$
H_{\alpha, V_{+}, V_{-}}-\xi_{0} \geq \lambda
$$

in the sense of quadratic forms, where $\lambda$ is a non-trivial and non-negative function $(c f .(2.15))$.

This Hardy inequality is called local since it reflects the local behaviour of the functions $V_{ \pm}$. In particular, if $V_{ \pm}$are compactly supported then $\lambda$ is compactly supported as a function of the first variable.

In any case, a global Hardy inequality follows by applying the classical Hardy inequality.

Theorem 5.2 (Global Hardy inequality). Assume that $V_{ \pm}$are non-negative, and that there exists $x_{1}^{0} \in \mathbb{R}$ and positive numbers $V_{0}$ and $R$ such that

$$
\forall x_{1} \in\left(x_{1}^{0}-R, x_{1}^{0}+R\right), \quad V_{+}\left(x_{1}\right) \geq V_{0} \quad \text { or } \quad V_{-}\left(x_{1}\right) \geq V_{0} .
$$

Then

$$
H_{\alpha, V_{+}, V_{-}}-\xi_{0} \geq c \rho \quad \text { with } \quad \rho(x):=\frac{1}{1+\left(x_{1}-x_{1}^{0}\right)^{2}}
$$

in the sense of quadratic forms. Here the constant $c$ depends on $V_{0}, R$ and $a$. 
Proof. The proof follows the strategy developed in [8, Sec. 3.3] to establish a similar global Hardy inequality in curved waveguides. For clarity of exposition, we divide the proof into several steps. Denote $I:=\left(x_{1}^{0}-R, x_{1}^{0}+R\right)$.

Step 1. The main ingredient in the proof is the classical one-dimensional Hardy inequality

$$
\forall \phi \in W_{0}^{1,2}\left(\mathbb{R}_{+}\right), \quad \int_{\mathbb{R}_{+}}\left|\phi^{\prime}(x)\right|^{2} \mathrm{~d} x \geq \frac{1}{4} \int_{\mathbb{R}_{+}} \frac{|\phi(x)|^{2}}{x^{2}} \mathrm{~d} x .
$$

We apply it in our case as follows. Let us define an auxiliary function $\eta: \mathbb{R} \rightarrow \mathbb{R}$ by $\eta\left(x_{1}\right):=\left|x_{1}-x_{1}^{0}\right| / R$ if $\left|x_{1}-x_{1}^{0}\right|<R$ and by setting it equal to 1 otherwise; we keep the same notation $\eta$ for the function $x \mapsto \eta\left(x_{1}\right)$ on $\mathbb{R}^{2}$. For any $\psi \in C_{0}^{\infty}\left(\mathbb{R}^{2}\right)$, let us write $\psi=\eta \psi+(1-\eta) \psi$. Applying the classical Hardy inequality to the function $\eta \psi$ and using Fubini's theorem we get

$$
\begin{aligned}
\int_{\mathbb{R}^{2}} \rho|\psi|^{2} & \leq 2 \int_{\mathbb{R}^{2}} \frac{|\eta \psi|^{2}}{\left(x_{1}-x_{1}^{0}\right)^{2}}+2 \int_{\mathbb{R}^{2}}|(1-\eta) \psi|^{2} \\
& \leq 16 \int_{\mathbb{R}^{2}}\left|\partial_{1} \eta\right|^{2}|\psi|^{2}+16 \int_{\mathbb{R}^{2}}|\eta|^{2}\left|\partial_{1} \psi\right|^{2}+2 \int_{I \times \mathbb{R}}|\psi|^{2} \\
& \leq 16 \int_{\mathbb{R}^{2}}\left|\partial_{1} \psi\right|^{2}+\left(2+16 / R^{2}\right) \int_{I \times \mathbb{R}}|\psi|^{2}
\end{aligned}
$$

By density, the inequality extends to all $\psi \in W^{1,2}=\mathfrak{D}\left(\mathcal{E}_{\alpha, V_{+}, V_{-}}\right)$.

Step 2. By Theorem 5.1, we have

$$
\mathcal{E}_{\alpha, V_{+}, V_{-}}[\psi]-\xi_{0}\|\psi\|_{L^{2}}^{2} \geq \underset{I \times \mathbb{R}}{\operatorname{essinf}} \lambda \int_{I \times \mathbb{R}}|\psi|^{2} \geq \lambda_{0} \int_{I \times \mathbb{R}}|\psi|^{2}
$$

for every $\psi \in \mathfrak{D}\left(\mathcal{E}_{\alpha, V_{+}, V_{-}}\right)$, where $\lambda_{0}:=\tilde{\lambda}\left(V_{0}, 0\right)=\tilde{\lambda}\left(0, V_{0}\right)$. Of course, $\lambda_{0}$ is a positive number under the stated hypotheses.

On the other hand, neglecting the non-negative potential term in (2.16), we have

$$
\mathcal{E}_{\alpha, V_{+}, V_{-}}[\psi]-\xi_{0}\|\psi\|_{L^{2}}^{2} \geq \int_{\mathbb{R}^{2}}\left|\partial_{1} \psi\right|^{2}
$$

for every $\psi \in \mathfrak{D}\left(\mathcal{E}_{\alpha, V_{+}, V_{-}}\right)$.

Step 3. Interpolating between the bounds (5.35) and (5.36), and using (5.34) in the latter, we arrive at

$$
\mathcal{E}_{\alpha, V_{+}, V_{-}}[\psi]-\xi_{0}\|\psi\|_{L^{2}}^{2} \geq \frac{\epsilon}{16} \int_{\mathbb{R}^{2}} \rho|\psi|^{2}+\left[(1-\epsilon) \lambda_{0}-\epsilon\left(\frac{1}{8}+\frac{1}{R^{2}}\right)\right] \int_{I \times \mathbb{R}}|\psi|^{2}
$$

for every $\psi \in \mathfrak{D}\left(\mathcal{E}_{\alpha, V_{+}, V_{-}}\right)$and $\epsilon \in(0,1)$. It is clear that the right hand side of this inequality can be made non-negative by choosing $\epsilon$ sufficiently small. Choosing $\epsilon$ 
such that the expression in the square brackets vanishes, the inequality yields the claim of the theorem with

$$
c:=\frac{\lambda_{0} / 16}{\lambda_{0}+1 / 8+1 / R^{2}} .
$$

It remains to observe that $\lambda_{0}$ depends on $V_{0}$ and $a$ through the definition (2.14).

As a direct consequence of Theorem 5.2, we get

Corollary 5.3. Assume the hypotheses of Theorem 5.2. Let $W$ be the operator in $L^{2}$ of multiplication by any bounded function $w$ for which there exists a positive constant $C$ such that $|w(x)| \leq C\left|x_{1}\right|^{-2}$ for a.e. $x \in \mathbb{R}^{2}$. Then there exists $\epsilon_{0}>0$ such that for every $\epsilon<\epsilon_{0}$,

$$
\inf \sigma\left(H_{\alpha, V_{+}, V_{-}}+\epsilon W\right) \geq \xi_{0} .
$$

Assume that $V_{ \pm}$vanish at infinity. Since also the potential $W$ of the corollary is bounded and vanishes at infinity, it is easy to see that the essential spectrum is not changed, i.e., $\sigma_{\text {ess }}\left(H_{\alpha, V_{+}, V_{-}}+\epsilon W\right)=\left[\xi_{0}, \infty\right)$, independently of the value of $\epsilon$ and irrespective of the signs of $V_{ \pm}$. It follows from the corollary that a certain critical value of $\epsilon$ is needed in order to generate discrete spectrum of $H_{\alpha, V_{+}, V_{-}}+\epsilon W$ if the Hardy inequality for $H_{\alpha, V_{+}, V_{-}}$exists. On the other hand, it is easy to see that $H_{\alpha, 0,0}+\epsilon W$ has eigenvalues below $\xi_{0}$ for arbitrarily small $\epsilon$ provided that $W$ is non-trivial and non-positive.

\section{$\S 6$. Resonances induced by broken symmetry}

As already stated (cf. Corollary 4.6), the Hamiltonian $H_{\alpha, V_{0}}$ of the system with mirror symmetry (2.26) admits embedded eigenvalues. In the following we show that breaking this symmetry by (2.27) will turn the eigenvalues into resonances.

The strategy we employ here is as follows. Our first aim is to show that the operator-valued function $z \mapsto R_{\alpha ; \epsilon}(z)$ has a second sheet analytic continuation in the following sense: for any $f, g \in C_{0}\left(\mathbb{R}^{2}\right)$ the operator $f R_{\alpha ; \epsilon}(z) g$ can be analytically continued to the lower half-plane as a bounded operator in $L^{2}$. Such a continuation will be denoted by $R_{\alpha ; \epsilon}^{I I}(\cdot) \equiv R_{\alpha ; \epsilon, f, g}^{I I}(\cdot)$. Of course, the above formulation implies that the function $z \mapsto\left(f, R_{\alpha ; \epsilon}(z) g\right)$ has a second sheet continuation. The analogous definition will be employed for the second sheet continuation of $R_{\alpha}(\cdot)$. To recover resonances in the system governed by $H_{\alpha ; \epsilon}$, we look for poles of $R_{\alpha ; \epsilon}^{I I}(\cdot)$. These poles are defined by the condition

$$
\operatorname{Ker} \Gamma^{I I}(z) \neq\{0\}, \quad \Im z<0,
$$

where $\Gamma^{I I}(\cdot)$ is the second sheet continuation of the analytic operator valuedfunction $z \mapsto \Gamma(z)$. Our aim is to find $z$ satisfying (6.37). 


\section{$\S 6.1$. The second sheet continuation of $\Gamma(\cdot)$}

Henceforth we assume

$$
V_{ \pm} \mathrm{e}^{C|x|} \in L^{\infty}(\mathbb{R}) \quad \text { for } \quad \text { some } \quad C>0
$$

The first auxiliary statement is contained in the following lemma.

Lemma 6.1. Suppose (6.38) holds. Then for any $i, j \in\{+,-\}$ and $z \in \mathbb{C} \backslash\left[\xi_{0}, \infty\right)$ the operator $\left|V_{i}\right|^{1 / 2} \mathrm{R}_{\alpha, i j}(z) V_{j}^{1 / 2}$ is Hilbert-Schmidt. Consequently, so is $B(z)$.

Proof. Using (2.21) we have

$$
\begin{aligned}
& \left|V_{i}\right|^{1 / 2} \mathrm{R}_{\alpha, i j}(z) V_{j}^{1 / 2} \\
& =\underbrace{\left|V_{i}\right|^{1 / 2} \mathrm{R}_{0, i j}(z) V_{j}^{1 / 2}}_{A_{1}}-\sum_{k, l \in\{+,-\}} \underbrace{\left|V_{i}\right|^{1 / 2} \mathrm{R}_{0, i k}(z)}_{A_{2}} \Gamma_{0, \alpha}(z)_{k l}^{-1} \underbrace{\mathrm{R}_{0, l j}(z) V_{j}^{1 / 2}}_{A_{3}} .
\end{aligned}
$$

It is well known that $R_{0}(z)$ is an integral operator:

$$
R_{0}(z) f(x)=\int_{\mathbb{R}^{2}} G_{0}(z ; x-w) f(w) \mathrm{d} w, \quad G_{0}(z ; x)=\frac{1}{2 \pi} K_{0}(\sqrt{z}|x|),
$$

where $K_{0}(\cdot)$ stands for the Macdonald function (cf. [1, Sec. 9.6]), $\Im \sqrt{z}>0$. Consequently, $\mathrm{R}_{0, i j}(z)$ is an integral operator with kernel $G_{0, i j}(z ; \cdot-\cdot)$ defined as the "bilateral" embedding of $G_{0}(z ; \cdot-\cdot)$ acting from $L_{j}^{2}$ to $L_{i}^{2}$. Using the properties of $K_{0}$ (see [1, eq. (9.6.8)]), we conclude that $G_{0}(z ; \cdot)$ has a logarithmic singularity at the origin and is continuous elsewhere; moreover it exponentially decays at infinity. This implies that $G_{0, i j}(z ; \cdot) \in L^{2}(\mathbb{R})$ and consequently $\left|G_{0, i j}(z ; \cdot)\right|^{2} \in L^{1}(\mathbb{R})$. Since $V_{ \pm} \in L^{\infty}(\mathbb{R}) \cap L^{1}(\mathbb{R})$ we have

$$
\begin{aligned}
\left\|\left|V_{i}\right|^{1 / 2} \mathrm{R}_{0, i j}(z) V_{j}^{1 / 2}\right\|_{\mathrm{HS}}^{2} & \leq \int_{\mathbb{R}^{2}}\left|V_{i}(x)\right|\left|G_{0, i j}(z ; x-y)\right|^{2}\left|V_{j}(y)\right| \mathrm{d} x \mathrm{~d} y \\
& \leq\left\|V_{j}\right\|_{\infty}\left\|\left|V_{i}\right|^{1 / 2} \mathrm{R}_{0, i j}(z)\right\|_{\mathrm{HS}}^{2} \\
& \leq\left\|V_{j}\right\|_{\infty}\left\|V_{i}\right\|_{L^{1}(\mathbb{R})}\left\|\left|G_{0, i j}(z)\right|^{2}\right\|_{L^{1}(\mathbb{R})}
\end{aligned}
$$

where $\|\cdot\|_{\text {HS }}$ denotes the Hilbert-Schmidt norm and the last step employs the Young inequality (cf. [25, Chap. IX.4, Ex. 1]). The above inequalities show that $A_{1}, A_{2}, A_{3}$ are Hilbert-Schmidt operators; note $\left\|\mathrm{R}_{0, i j}(z) V_{j}^{1 / 2}\right\|_{\mathrm{HS}}=$ $\left\|\left|V_{j}\right|^{1 / 2} \mathrm{R}_{0, j i}(z)\right\|_{\mathrm{HS}}$. Moreover, since the $\Gamma_{0, \alpha}(z)_{k l}^{-1}$ are bounded, the operators $\left|V_{i}\right|^{1 / 2} \mathrm{R}_{\alpha, i j}(z) V_{j}^{1 / 2}$ are Hilbert-Schmidt as well.

Remark 6.2. Note that to prove the above lemma we use only $V_{ \pm} \in L^{\infty}(\mathbb{R}) \cap$ $L^{1}(\mathbb{R})$; the stronger assumption (6.38) will be used in the following. 
Suppose $\mathcal{B}$ is an open subset of $\left[\xi_{0}, \infty\right)$ and $E_{\mathcal{B}}$ denotes the spectral measure of $H_{\alpha, V_{+}, V_{-}}$. Denote $\mathcal{H}_{\mathrm{ac}}:=\left\{\psi \in L^{2}: \mathcal{B} \mapsto\left(\psi, E_{\mathcal{B}} \psi\right)\right.$ is absolutely continuous $\}$.

Lemma 6.3. Suppose (6.38) holds. There is a finite set $\xi \subset \mathbb{R}$ such that:

(i) For any interval $[a, b] \subset\left[\xi_{0}, \mu_{0}\right]$ which is disjoint from $\mathcal{E}$ we have $\operatorname{Ran} E_{(a, b)}$ $\subset \mathcal{H}_{\mathrm{ac}}$.

(ii) There exists a region $\Omega_{-} \subset \mathbb{C}_{-}$with boundary containing $\left(\xi_{0}, \mu_{0}\right)$ and an operator-valued function $R_{\alpha, V_{+}, V_{-}}^{I I}(\cdot)$ analytic in $\Omega \backslash \mathcal{E}$, where $\Omega=\mathbb{C}_{+} \cup$ $\left(\xi_{0}, \mu_{0}\right) \cup \Omega_{-}$, which is the analytic continuation of $R_{\alpha, V_{+}, V_{-}}(\cdot)$.

Proof. The operator $R_{\alpha}(\cdot)$ is analytic in $\mathbb{C} \backslash\left[\xi_{0}, \infty\right)$. The Stone formula implies that $\operatorname{s}^{-\lim _{\varepsilon \rightarrow 0}}\left(R_{\alpha}(\lambda+i \varepsilon)-R_{\alpha}(\lambda-i \varepsilon)\right) \neq 0$ for $\lambda \in\left(\xi_{0}, \mu_{0}\right)$. In the following we write $R_{\alpha}(\lambda \pm i 0)$ for s-lim $\lim _{\varepsilon \rightarrow 0} R_{\alpha}(\lambda \pm i \varepsilon)$ and analogously for resolvents of other operators.

Our first aim is to show that $R_{\alpha}(\cdot)$ can be analytically continued from $\mathbb{C}_{+}$ through $\left(\xi_{0}, \mu_{0}\right)$ to the lower half-plane in the sense described at the beginning of this section. Recall

$$
R_{\alpha}(z)=R_{\alpha}^{c}(z)+R^{d}(z), \quad R^{d}(z)=\sum_{j \in \mathcal{N}} R_{\alpha}^{j, d}(z) .
$$

Note that $R_{\alpha}^{c}(z)$ is analytic for $z \in \mathbb{C} \backslash[0, \infty)$ (cf. (2.18)). Furthermore, if $\alpha a>1$ then the component $R_{\alpha}^{1, d}(z)$ of $R_{\alpha}^{d}(z)$ is analytic for $z \in \mathbb{C} \backslash\left[\xi_{1}, \infty\right)$. On the other hand, the analytic continuation of $R_{\alpha}^{0, d}(\cdot)$ through $\left(\xi_{0}, \infty\right)$ is defined by the second sheet values of $\tau_{0}$, i.e. $\Im \tau_{0}^{I I} \leq 0$. More precisely, for $f, g \in C_{0}\left(\mathbb{R}^{2}\right)$ the operator $f R_{\alpha}^{0, d}(\cdot) g$ has a second sheet analytical continuation which is an integral operator with kernel $f(x) G_{\alpha}^{0, d, I I}(z ; x-y) g(y)$ and $G_{\alpha}^{0, d, I I}$ is defined by $(2.17)$, with $\tau_{0}$ replaced by $\tau_{0}^{I I}$. The resulting operator is denoted by $R_{\alpha}^{0, d, I I}(\cdot)$. Consequently, we define the second sheet continuation of $R_{\alpha}(z)$ as $R_{\alpha}^{I I}(z)=R_{\alpha}^{c}(z)+R_{\alpha}^{d, I I}(z)$ where $R_{\alpha}^{d, I I}(z)=R_{\alpha}^{0, d, I I}(z)+R_{\alpha}^{1, d}(z)$ and $z \in \mathbb{C}_{+} \cup\left(\xi_{0}, \mu_{0}\right) \cup \mathbb{C}_{-}$. The operator $R_{\alpha}^{I I}(z)$ is analytic bounded in $L^{2}$ in the sense described at the beginning of the section.

By means of $G_{\alpha}^{0, d, I I}(z ; x-y)$ we define the operator $\left|V_{i}\right|^{1 / 2} \mathrm{R}_{\alpha, i j}^{0, d I}(z) V_{j}^{1 / 2}$ : $L_{j}^{2} \rightarrow L_{i}^{2}, i, j \in\{+,-\}$, with

$$
\begin{aligned}
& \left\|\left|V_{i}\right|^{1 / 2} \mathrm{R}_{\alpha, i j}^{0, d, I I}(z) V_{j}^{1 / 2}\right\|_{\mathrm{HS}}^{2} \\
& \quad=\frac{\left|\phi_{0}(i a) \phi_{0}(j a)\right|^{2}}{4\left|\tau_{0}^{I I}(z)\right|^{2}} \int_{\mathbb{R}^{2}} \mathrm{e}^{-C\left(\left|x_{1}\right|+\left|y_{1}\right|\right)} \mathrm{e}^{-2 \Im \tau_{0}^{I I}(z)\left|x_{1}-y_{1}\right|}\left|h_{i}\left(x_{1}\right) h_{j}\left(y_{1}\right)\right| \mathrm{d} x_{1} \mathrm{~d} y_{1},
\end{aligned}
$$

where $h_{ \pm}:=V_{ \pm} \mathrm{e}^{C|\cdot|} \in L^{\infty}(\mathbb{R})$ (cf. (6.38)). The above expression is finite if $\Im \tau_{0}^{I I}(z)>-C / 2$. For $\left|V_{i}\right|^{1 / 2} \mathrm{R}_{\alpha, i j}^{1, d}(z) V_{j}^{1 / 2}$ the analogous expression is always finite because $R_{\alpha}^{1, d}(z)$ is analytic on $\mathbb{C}_{+} \cup\left(\xi_{0}, \mu_{0}\right) \cup \mathbb{C}_{-}$, and $\Im \tau_{1}(z)>0$. 
Furthermore, since $\left|V_{i}\right|^{1 / 2} \mathrm{R}_{\alpha, i j}(z) V_{j}^{1 / 2}$ for $z \in \mathbb{C} \backslash\left[\xi_{0}, \infty\right)$ is a HilbertSchmidt operator (cf. Lemma 6.1), as also is $\left|V_{i}\right|^{1 / 2} \mathrm{R}_{\alpha, i j}^{d}(z) V_{j}^{1 / 2}$, we conclude that $\left|V_{i}\right|^{1 / 2} \mathrm{R}_{\alpha, i j}^{c}(z) V_{j}^{1 / 2}$ is a Hilbert-Schmidt operator with boundary values $\left|V_{i}\right|^{1 / 2} \mathrm{R}_{\alpha, i j}^{c}(\lambda \pm i 0) V_{j}^{1 / 2}, \lambda \in\left(\xi_{0}, \mu_{0}\right)$, being compact. Consequently, $B_{i j}(\lambda+i 0)$, $\lambda \in\left(\xi_{0}, \mu_{0}\right)$, is compact and $B_{i j}(\cdot)$ has second sheet continuation $B_{i j}^{I I}(\cdot)=$ $\left|V_{i}\right|^{1 / 2} \mathrm{R}_{\alpha, i j}^{I I}(\cdot) V_{j}^{1 / 2}$ through $\left(\xi_{0}, \mu_{0}\right)$. Finally, we conclude that $B^{I I}(z)$ is compact for $z \in \Omega=\mathbb{C}_{+} \cup\left(\xi_{0}, \mu_{0}\right) \cup \Omega_{-}$, where $\Omega_{-}$is a region in $\mathbb{C}_{-}$with boundary containing $\left(\xi_{0}, \mu_{0}\right)$ and determined by the condition $\Im \tau_{0}(z)>-C / 2$.

Note that the operator $f \check{R}_{\alpha, i}(z) V_{i}^{1 / 2}$, where $f \in C_{0}\left(\mathbb{R}^{2}\right)$, has an analytic second sheet continuation. Indeed, as the above discussion shows, the only nontrivial component is given by $f \check{R}_{\alpha, i}^{0, d}(z) V_{i}^{1 / 2}$. Since its Hilbert-Schmidt norm is

$$
\begin{aligned}
& \left\|f \check{R}_{\alpha, i}^{0, d I}(z) V_{i}^{1 / 2}\right\|_{\mathrm{HS}}^{2} \\
& \quad=\frac{\left|\phi_{0}(i a)\right|^{2}}{4\left|\tau_{0}^{I I}(z)\right|^{2}} \int_{\mathbb{R}^{2}} \int_{\mathbb{R}} \mathrm{e}^{-C\left|y_{1}\right|} \mathrm{e}^{-2 \Im \tau_{0}^{I I}(z)\left|x_{1}-y_{1}\right|}|f(x)|^{2}\left|h_{i}\left(y_{1}\right)\right| \mathrm{d} x \mathrm{~d} y_{1},
\end{aligned}
$$

where $x=\left(x_{1}, x_{2}\right)$ and $h_{i}:=V_{i} \mathrm{e}^{C|\cdot|} \in L^{\infty}(\mathbb{R})$ we conclude that $f \check{R}_{\alpha, i}^{0, d I}(z) V_{i}^{1 / 2}$ is analytic for $z \in \Omega$ and bounded as an operator acting from $L_{i}^{2}$ to $L^{2}$. The analogous statement can be obtained for $\left|V_{i}\right|^{1 / 2} \hat{R}_{\alpha, i}(z) f$.

(i) Since the condition $\operatorname{Ker}[1+B(z)] \neq\{0\}$ determines the poles of $R_{\alpha, V_{+}, V_{-}}(\cdot)$ (cf. (2.25)), which is the resolvent of a self-adjoint operator, the former has no solution for $z \in \mathbb{C}_{+}$. Combining compactness of $B(z)$ and the analytic Fredholm theorem (see, e.g., [24, Thm. VI.14]) with the fact that $z \mapsto B(z)$ is analytic for $z \in$ $\mathbb{C}_{+} \cup\left(\xi_{0}, \mu_{0}\right)$, we conclude that the operator $[1+B(z)]^{-1}$ exists and it is bounded analytic in $z \in \mathbb{C}_{+}$with boundary values $z=\lambda+i 0$, provided $\lambda \in\left(\xi_{0}, \mu_{0}\right)$ avoids a finite set $\mathcal{E}$ of real numbers; for an analogous discussion see [26, Thm. XIII.21]. Moreover, the operator $|f| R_{\alpha}(\lambda+i \varepsilon)|f|, f \in C_{0}\left(\mathbb{R}^{2}\right)$, is uniformly bounded for $0<\varepsilon<1$ and $\lambda \in\left(\xi_{0}, \mu_{0}\right)$. The operators $|f| \check{R}_{\alpha, i}(z) V_{i}^{1 / 2}$ and $\left|V_{i}\right|^{1 / 2} \hat{R}_{\alpha, i}(z)|f|$ are uniformly bounded as well. Combining the above statements with the resolvent formula (2.23), we come to the conclusion that, for any $f \in C_{0}\left(\mathbb{R}^{2}\right)$, the function

$$
\begin{aligned}
|f| R_{\alpha, V_{+}, V_{-}}(z)|f|= & |f| R_{\alpha}(z)|f| \\
& -\sum_{i, j \in\{+,-\}}\left(|f| \check{R}_{\alpha, i}(z) V_{i}^{1 / 2}\right)[1+B(z)]_{i j}^{-1}\left(\left|V_{j}\right|^{1 / 2} \hat{R}_{\alpha, j}(z)|f|\right)
\end{aligned}
$$

is uniformly bounded with respect to $z=\lambda+i \varepsilon, 0<\varepsilon<1, \lambda \in[a, b]$. Furthermore, since

$$
\left|\left(g, R_{\alpha, V_{+}, V_{-}}(z) g\right)\right| \leq\left\||g|^{1 / 2}\right\|^{2}\left\||g|^{1 / 2} R_{\alpha, V_{+}, V_{-}}(z)|g|^{1 / 2}\right\|
$$


for any $g \in C_{0}\left(\mathbb{R}^{2}\right)$, the assumption of [26, Thm. XIII.19] is fulfilled, which yields the claim. (Note that in the last expression the same notation $\|\cdot\|$ was used for the operator norm and for the norm of a function.)

(ii) Using again the Fredholm theorem and the compactness of $B^{I I}(\cdot)$, we find that the operator $\left[1+B^{I I}(z)\right]^{-1}$ exists and it is bounded analytic in $\Omega \backslash \mathcal{E}$. For $z \in \Omega \backslash \mathcal{E}$ we construct $R_{\alpha, V_{+}, V_{-}}^{I I}(z)$ as

$$
R_{\alpha, V_{+}, V_{-}}^{I I}(z)=R_{\alpha}^{I I}(z)-\check{R}_{\alpha}^{I I}(z) \mathrm{V}^{1 / 2}\left[1+B^{I I}(z)\right]^{-1}|\mathrm{~V}|^{1 / 2} \hat{R}_{\alpha}^{I I}(z)
$$

(cf. (2.24)), where $\check{R}_{\alpha}^{I I}(z) \mathrm{V}^{1 / 2}$ and $\hat{R}_{\alpha}^{I I}(z)|\mathrm{V}|^{1 / 2}$ are the second sheet continuations already discussed.

Corollary 6.4. $\sigma_{\mathrm{sc}}\left(H_{\alpha, V_{+}, V_{-}}\right) \cap\left[\xi_{0}, \mu_{0}\right]=\emptyset$.

Proof. It follows from the previous theorem that $\sigma_{\mathrm{sc}}\left(H_{\alpha, V_{+}, V_{-}}\right) \cap\left[\xi_{0}, \mu_{0}\right] \subset \mathcal{E}$ is a finite set; this implies the claim.

With no danger of confusion, we employ the notation $\lambda \mapsto E(\lambda)$ for the spectral resolution of $H_{\alpha, V_{0}}$ for $\lambda \in\left[\xi_{0}, \infty\right) \backslash \mathcal{E}$. Then the operator $R_{\alpha, V_{0}}(z)$ admits the decomposition

$$
R_{\alpha, V_{0}}(z)=\sum_{i=1}^{N} \frac{1}{\nu_{i}-z} P_{i}+\int_{\left[\xi_{0}, \infty\right)} \frac{\mathrm{d} E(\lambda)}{\lambda-z},
$$

where $\left\{\nu_{i}\right\}_{i=1}^{N}=\sigma_{\mathrm{p}}\left(H_{\alpha, V_{0}}\right), P_{i}=\omega_{i}\left(\omega_{i}, \cdot\right)$, and $\omega_{i}$ are the corresponding eigenvectors. From the definition of $E(\cdot)$ and Corollary 6.4, we conclude that $\left(\xi_{0}, \mu_{0}\right) \ni$ $\lambda \mapsto E(\lambda)$ projects onto $\mathcal{H}_{\mathrm{ac}}$. Given $f, g \in L^{2}$, let us denote by $F(\cdot)_{f, g}$ the RadonNikodym derivative of $\left(\xi_{0}, \mu_{0}\right) \ni \lambda \mapsto(f, E(\lambda) g)$. The limit of $z=\gamma+i \varepsilon$ as $\varepsilon \rightarrow 0$ with $\gamma \in\left(\xi_{0}, \mu_{0}\right) \backslash \mathcal{E}$ and $f, g \in C_{0}\left(\mathbb{R}^{2}\right)$ takes the form

$$
\begin{aligned}
\left(f, R_{\alpha, V_{0}}(\gamma+i 0) g\right)= & \sum_{i=1}^{N} \frac{1}{\nu_{i}-\gamma}\left(f, \omega_{i}\right)\left(\omega_{i}, g\right)+\mathcal{P} \int_{\left[\xi_{0}, \mu_{0}\right)} \frac{F_{f, g}(\lambda) \mathrm{d} \lambda}{\lambda-\gamma} \\
& +\int_{\mu_{0}}^{\infty} \frac{\mathrm{d}(f, E(\lambda) g)}{\lambda-\gamma}+\pi i F_{f, g}(\gamma),
\end{aligned}
$$

where $\mathcal{P}$ denotes principal value. By the edge-of-the-wedge theorem (cf. [27]), we get

$$
\left(f, R_{\alpha, V_{0}}(\gamma+i 0) g\right)=\left(f, R_{\alpha, V_{0}}^{I I}(\gamma-i 0) g\right), \quad f, g \in C_{0}\left(\mathbb{R}^{2}\right),
$$

where $R_{\alpha, V_{0}}^{I I}(\cdot)$ denotes the second sheet continuation of $R_{\alpha, V_{0}}(\cdot)$ given in Lemma 6.3. The operator $R_{\alpha, V_{0}}(\cdot)$ is the resolvent of the mirror symmetry system. 
Now we introduce the potential $V_{\epsilon}=\epsilon V_{p}$ living on $\mathbb{R} \times\{+a\}$. By means of $R_{\alpha, V_{0}}^{I I}(z)$ we determine

$$
\Gamma^{I I}(z)=1+\left|V_{\epsilon}\right|^{1 / 2} \mathrm{R}_{\alpha, V_{0}}^{I I}(z) V_{\epsilon}^{1 / 2}, \quad z \in \Omega \backslash \mathcal{E}
$$

where the last term is the analytic second sheet continuation of the operator $\left|V_{\epsilon}\right|^{1 / 2} \mathrm{R}_{\alpha, V_{0}}(z) V_{\epsilon}^{1 / 2}: L_{+}^{2} \rightarrow L_{+}^{2}$.

\section{$\S 6.2$. Zeros of $\Gamma^{I I}(\cdot)$; the Fermi golden rule}

Henceforth we assume that the set of embedded eigenvalues of $H_{\alpha, V_{0}}$ is not empty (this is true, for instance, under the hypotheses of Corollary 4.6). Then there exists an integer $k_{0} \leq N$ such that for all $i \geq k_{0}$ we have $\nu_{i} \in\left(\xi_{0}, \mu_{0}\right)$. Given $k \geq k_{0}$, define

$$
A_{k}(z):=R_{\alpha, V_{0}}(z)-\frac{1}{\nu_{k}-z} P_{k}
$$

Analogously we define $A_{k}^{I I}(z)$ replacing $R_{\alpha, V_{0}}(z)$ in $(6.44)$ by $R_{\alpha, V_{0}}^{I I}(z)$.

The main result of this section is the following theorem.

Theorem 6.5. Suppose (6.38) holds. Assume that $\nu_{k} \in\left(\xi_{0}, \mu_{0}\right)$ is an embedded eigenvalue of $H_{\alpha, V_{0}}$. Then the resolvent of $H_{\alpha ; \epsilon}$ has a pole $z_{k}$ satisfying

$$
z_{k}=\nu_{k}+\epsilon\left(\omega_{k}, V_{p} \omega_{k}\right)_{L_{+}^{2}}+\epsilon^{2}\left(\Gamma_{r}+i \Gamma_{i}\right)+\mathcal{O}\left(\epsilon^{3}\right) \quad \text { as } \epsilon \rightarrow 0
$$

where

$$
\begin{aligned}
& \Gamma_{r}:=-\sum_{i \in\{1, \ldots, N\} \backslash k} \frac{1}{\nu_{i}-\nu_{k}}\left|\left(\omega_{k}, V_{p} \omega_{i}\right)_{L_{+}^{2}}\right|^{2}-\mathcal{P} \int_{\left[\xi_{0}, \infty\right)} \frac{\mathrm{d}\left(\omega_{k}, V_{p} E(\lambda) V_{p} \omega_{k}\right)_{L_{+}^{2}}}{\lambda-\nu_{k}}, \\
& \Gamma_{i}:=-\pi F_{+}\left(\nu_{k}\right) \text { with } \quad F_{+}\left(\nu_{k}\right):=\left.\frac{\mathrm{d}}{\mathrm{d} \nu}\left(\omega_{k}, V_{p} E(\nu) V_{p} \omega_{k}\right)_{L_{+}^{2}}\right|_{\nu=\nu_{k}} .
\end{aligned}
$$

(Here the functions $\omega_{k}$ are understood as embeddings into $L_{+}^{2}$. Similarly, E $(\cdot)$ is a family of operators acting from $L_{+}^{2}$ to $L_{+}^{2}$.)

Proof. Note that the function $z \mapsto\left(f, A_{k}^{I I}(z) g\right), f, g \in C_{0}\left(\mathbb{R}^{2}\right)$, is analytic in a neighbourhood $M$ of $\nu_{k}$. Furthermore, for $\epsilon$ small enough, say $\epsilon \leq \epsilon_{0}$, where $\epsilon_{0}>0$, the operator $G_{\epsilon, k}(z):=1+\left|V_{\epsilon}\right|^{1 / 2} A_{k}^{I I}(z) V_{\epsilon}^{1 / 2}$ is invertible. We define the function $\eta_{k}:\left[0, \epsilon_{0}\right) \times M \rightarrow \mathbb{C}$ by

$$
\eta_{k}(\epsilon, z):=z-\nu_{k}-\left(\omega_{k}, V_{\epsilon}^{1 / 2} G_{\epsilon, k}(z)^{-1}\left|V_{\epsilon}\right|^{1 / 2} \omega_{k}\right)_{L_{+}^{2}} .
$$


Suppose $z \in M \backslash \nu_{k}$. A straightforward calculation using (6.43) and (6.44) shows that $\phi \in \operatorname{Ker} \Gamma^{I I}(z)$ if, and only if,

$$
\phi+\frac{1}{\nu_{k}-z}\left(\omega_{k}, V_{\epsilon}^{1 / 2} \phi\right) G_{\epsilon, k}(z)^{-1}\left|V_{\epsilon}\right|^{1 / 2} \omega_{k}=0 .
$$

This means that $\operatorname{Ker} \Gamma^{I I}(z) \neq\{0\}$ if, and only if, $z$ is a solution of

$$
\eta_{k}(\epsilon, z)=0 \text {. }
$$

After expanding $G_{\epsilon, k}(z)^{-1}$ with respect to $\epsilon$, the function $\eta(\epsilon, z)$ reads

$$
\eta_{k}(\epsilon, z)=z-\nu_{k}-\epsilon\left(\omega_{k}, V_{p} \omega_{k}\right)_{L_{+}^{2}}+\epsilon^{2}\left(\omega_{k}, V_{p} A_{k}^{I I}(z) V_{p} \omega_{k}\right)_{L_{+}^{2}}+\mathcal{O}\left(\epsilon^{3}\right) .
$$

The function $\eta_{k}$ is analytic in $z \in M$ and it is $C^{1}$ in both variables. It is clear that $\eta_{k}\left(0, \nu_{k}\right)=0$ and $\partial_{z} \eta_{k}\left(0, \nu_{k}\right)=1$. Applying the implicit function theorem to (6.45) and using (6.46), we find that there exists an open neighbourhood $\mathcal{U}_{0} \subset \mathbb{R}_{+}$of zero and a unique function $z_{k}: \mathcal{U}_{0} \rightarrow \mathbb{C}$ of $\epsilon$ given by

$$
z_{k}=\nu_{k}+\epsilon\left(\omega_{k}, V_{p} \omega_{k}\right)_{L_{+}^{2}}-\epsilon^{2}\left(\omega_{k}, V_{p} A_{k}^{I I}\left(\nu_{k}-i 0\right) V_{p} \omega_{k}\right)_{L_{+}^{2}}+\mathcal{O}\left(\epsilon^{3}\right),
$$

and being a zero of $\Gamma^{I I}(\cdot)$. Employing $(6.44),(6.41)$ and $(6.42)$, we get the statement of the theorem.

The above results can be summarized as follows. Suppose the mirror symmetry system (2.26) has an embedded eigenvalue $\nu$. Once we break the symmetry by introducing the "perturbant" $V_{\epsilon}=\epsilon V_{p}$, the pole of the resolvent shifts from the spectrum and makes the second sheet continuation a pole of the resolvent. The imaginary component of the pole is related to the resonance width $\Gamma_{w}:=-2 \Im z_{k}=$ $\mathcal{O}\left(\epsilon^{2}\right)$. This means that for $\epsilon$ small the resonance is physically essential. Employing the lowest order perturbation in $\epsilon$, we can write

$$
\left.\Gamma_{w} \approx 2 \pi \frac{\mathrm{d}\left(\omega_{k}, V_{p} \mathrm{E}(\lambda) V_{p} \omega_{k}\right)_{L_{+}^{2}}}{\mathrm{~d} \lambda}\right|_{\lambda=\nu_{k}} \epsilon^{2} .
$$

This gives the Fermi golden rule. Moreover, $\Gamma_{w}^{-1}$ determines the life time of the resonance state.

Remark 6.6. Resonance phenomena induced by broken symmetry were studied in $[13,20]$. However, in the models analyzed there, the singular potentials are constants and the broken symmetry has a rather geometrical character. The methods derived in this paper essentially differ from the technics applied in [13, 20]. Finally, let us mention that the resonance phenomena and the decay law were recently reviewed in [10]. Systems with singular potentials were considered as examples of solvable models. 


\section{Acknowledgements}

The authors are grateful to the anonymous referee for a careful reading of the manuscript, for pointing out an error contained in the first version of the paper and for many other valuable remarks and suggestions. The work has been partially supported by RVO61389005 and the GACR grant No. P203/11/0701.

\section{References}

[1] M. Abramowitz and I. Stegun, Handbook of Mathematical Functions, 1972. Zbl 0543.33001

[2] S. Albeverio, F. Gesztesy, R. Høegh-Krohn, and H. Holden, Solvable models in quantum mechanics, 2nd printing, Amer. Math. Soc., Providence, RI, 2005. Zbl 1078.81003 MR 2105735

[3] S. Albeverio and P. Kurasov, Singular perturbations of differential operators, Cambridge Univ. Press, 2000. Zbl 0945.47015 MR 1752110

[4] J. Blank, P. Exner, and M. Havlíček, Hilbert space operators in quantum physics, 2nd ed., Springer, 2008. Zbl 1163.47060 MR 2458485

[5] J. F. Brasche, P. Exner, Yu. A. Kuperin, and P. Šeba, Schrödinger operators with singular interactions, J. Math. Anal. Appl. 184 (1994), 112-139. Zbl 0820.47005 MR 1275948

[6] J. F. Brasche and A. Teta, Spectral analysis and scattering theory for Schrödinger operators with an interaction supported by a regular curve, in Ideas and methods in quantum and statistical physics (Oslo, 1988), Cambridge Univ. Press, 1992, 197-211. Zbl 0787.35057 MR 1190526

[7] Y. Dermenjian, M. Durand, and V. Iftimie, Spectral analysis of an acoustic multistratified perturbed cylinder, Comm. Partial Differential Equations 23 (1998), 141-169. Zbl 0907.47046 MR 1608508

[8] T. Ekholm, H. Kovařík, and D. Krejčiřík, A Hardy inequality in twisted waveguides, Arch. Ration. Mech. Anal. 188 (2008), 245-264. Zbl 1167.35026 MR 2385742

[9] P. Exner, Leaky quantum graphs: a review, in Analysis on graphs and its applications (Cambridge, 2007), P. Exner et al. (ed.), Proc. Sympos. Pure Math. 77, Amer. Math. Soc., Providence, RI, 2008, 523-564. Zbl 1153.81487 MR 2459890

[10] Solvable models of resonances and decays, in Advances in partial differential equations, Oper. Theory Adv. Appl. 232, Birkhäuser, 2013, 165-227. Zbl 1266.81094

[11] P. Exner and T. Ichinose, Geometrically induced spectrum in curved leaky wires, J. Phys. A 34 (2001), 1439-1450. Zbl 1002.81024 MR 1819942

[12] P. Exner and S. Kondej, Curvature-induced bound states for a $\delta$ interaction supported by a curve in $\mathbb{R}^{3}$, Ann. Henri Poincaré 3 (2002), 967-981. Zbl 1017.81007 MR 1937610

[13] Schrödinger operators with singular interactions: a model of tunnelling resonances, J. Phys. A 37 (2004), 8255-8277. Zbl 1072.81056 MR 2092796

[14] Scattering by local deformations of a straight leaky wire, J. Phys. A 38 (2005), 4865-4874. Zbl 1071.81027 MR 2148629

[15] P. Exner and K. Yoshitomi, Asymptotics of eigenvalues of the Schrödinger operator with a strong delta-interaction on a loop, J. Geom. Phys. 41 (2002), 344-358. Zbl 1083.35034 MR 1888470

[16] _ Persistent currents for the $2 D$ Schrödinger operator with a strong $\delta$-interaction on a loop, J. Phys. A 35 (2002), 3479-3487. Zbl 1010.81012 MR 1907374 
[17] P. Freitas and D. Krejčiř́ik, Waveguides with combined Dirichlet and Robin boundary conditions, Math. Phys. Anal. Geom. 9 (2006), 335-352. Zbl 1151.35061 MR 2329432

[18] I. S. Gradshteyn and I. M. Ryzhik, Tables of integrals, series, and products, 7th ed., Academic Press, NY, 2007. Zbl 1208.65001 MR 2360010

[19] T. Kato, Pertubation theory for linear operators, Springer, Berlin, 1980. Zbl 0836.47009 MR 1335452

[20] S. Kondej, Resonances induced by broken symmetry in a system with a singular potential, Ann. Henri Poincaré 13 (2012), 1451-1467. Zbl 1252.81076 MR 2966468

[21] D. Krejčiřík and J. Kř́ž, On the spectrum of curved quantum waveguides, Publ. RIMS Kyoto Univ. 41 (2005), 757-791. Zbl 1113.35143 MR 2154341

[22] A. Posilicano, A Krein-like formula for singular perturbations of self-adjoint operators and applications, J. Funct. Anal. 183 (2001), 109-147. Zbl 0981.47022 MR 1837534

[23] _ Boundary triples and Weyl functions for singular perturbations of self-adjoint operators, Methods Funct. Anal. Topol. 10 (2004), 57-63. Zbl 1066.47024 MR 2067952

[24] M. Reed and B. Simon, Methods of modern mathematical physics: I. Functional analysis, Academic Press, New York, 1972. Zbl 0242.46001 MR 0493419

[25] L Methods of modern mathematical physics: II. Fourier analysis. Self-adjointness, Academic Press, New York, 1975. Zbl 0308.47002 MR 0493420

[26] _ Methods of modern mathematical physics: IV. Analysis of operators, Academic Press, New York, 1978. Zbl 0401.47001 MR 0493421

[27] W. Rudin, Lectures on the edge-of-the-wedge theorem, CBMS Reg. Conf. Ser. Math. 6, Amer. Math. Soc., 1971. Zbl 0214.09001 MR 0310288

[28] P. Stollmann and J. Voigt, Perturbation of Dirichlet forms by measures, Potential Anal. 5 (1996), 109-138. Zbl 0861.31004 MR 1378151 